\title{
Ueber den Einfluss von Trigeminus-Reizen auf die Sinnesempfindungen, insbesondere auf den Gesichtssinn.
}

Von

\section{Victor Urbantschitsch}

in Wien.

An mehreren Patienten mit chronischem Catarrh des Mittelohres fiel mir der bedeutende Einfluss auf, der vom Ohre aus auf das Sehvermögen ausgeübt werden kann.

Der erste Fall betraf einen 70 jährigen Mann, der durch einige Monate vor dem Eintritt in meine Behandlung gleichzeitig mit der Verminderung des Gehörs eine Verdunkelung des Gesichtsfeldes mit einer auffälligen Abnahme des Sehvermögens erlitten hatte; während der Einfiihrung einer Bougie durch den geschwellten Tubenkanal in die Paukenhöhle, erfolgte plötzlich eine Aufhellung des Gesichtsfeldes und eine bedeutende Sehverbesserung, welche auch gegenwärtig, seit einem Jahre, unverändert geblieben ist. In einem zweiten Falle war eine mehrmonatliche Schwachsichtigkeit im Verlauf der Ohrenbehandlung allmählich zurïckgegangen. Bei einem dritten Patienten hatte eine zweimalige Bougirung der Ohrtrompete eine beträchtliche Steigerung der Sehfunktion herbeigeführt, die sich nunmehr, seit 6 Monaten, constant erhält. Ein vierter Fall endlich betrifft eine Patientin, welche am linken Ohr von heftigen neuralgischen Anfällen (Otalgia tympanica) zeitweise befallen wurde und dabei eine solche Verdunklung des Gesichtssinnes erfuhr, dass Patientin während dieser Zeit weder lesen noch weibliche Handarbeiten verrichten konnte; nach dem Anfalle kehrte das frühere Sehvermögen wieder zurück.

Bei Durchsicht der Literatur über diesen Gegenstand fand ich nur eine diesbezigliche Bemerkung Delea $u^{\prime} \mathrm{s}^{1}$ ) aus dem Jahre

1) Journ. d. connaissances médico-chir. 1838, Nr. 6, ref. in den Med. Jahrb., Wien 1840, Bd. 23, p. 315. 
1838 vor, welcher Autor in zwei Fällen von einseitiger eitriger Entzïndung der Paukenhöhle eine Abnahme des Sehvermögens am Auge der kranken Seite beobachtete, die sich in dem einen Falle nach einer antiphlogistischen Behandinng, in dem anderen Falle ohne eine solche, mit der Besserung des Ohrenleidens wieder zuriickgebildet hatte.

Da ich aus den angefiubrten Fällen ersah, dass der bisher vollständig unberücksichtigt gebliebene Einfluss einer Erkrankung des Gehörorgans auf das Sehvermögen keineswegs als AusnahmsErscheinung auftritt, so stellte ich zunächst an einer Reihe von Ohrenkranken einschlägige Untersuchungen in der Weise an, dass bei jedem Versuchsindividum am Beginne der Behandlung und ferner in den verschiedenen Stadien der Ohrenerkrankung wiederholt Sehpriufungen vorgenommen wurden.

Sämmtliche Versuche fanden selbstrerständlich unter gleichen äusseren Bedingungen statt: Die Sehprifungen erfolgten unter $A b-$ schluss des Tageslichtes bei künstlicher Beleuchtung, bei stets gleicher Lichtintensität and constanter Entfernung des Lichtes und des Versuchsindividums rom Sehobjekte. Die Versuchsperson hatte vor der Vornahme der Sehproben in dem Untersuchungsraume durch mindestens כ̌ Minuten zu verweilen. Da es sich bei meinen Versuchen nur um die Bestimmung des Sehvermögens in einer gegebenen Distanz bandelte, so habe ich von allen Prifungen auf die Sehschärfe und auf die Refraction ganz abgesehen und mich nur darauf beschränkt, das Sehvermögen des Individuums dadurch festzustellen, dass ich den kleinsten Druck ermittelte, der in einer bestimmten Distanz uiberhaupt wahrgenommen wurde. $\mathrm{Zu}$ diesem Zwecke wurden von den Ja ge r'schen Schriftskalen, welche ich bei meinen Versuchen benitzte, stets jene Nr. zum Ausgangspunkt der Untersuchung gewählt, bei dem die Versuchsperson nur einzelne Buchstaben oder Silben lesen konnte.

Das Ergebniss der Sehprüfungen zeigt sich nämlich bei einzelnen Individuen sebr verschieden je nachdem man von einer für die Versuchsperson deutlich lesbaren Schriftskala zu einer nur theilweise wahrnehmbaren übergeht oder aber umgekehrt, mit einer für das Auge des Untersuchten zu niederen Skala beginnt und allmählich bis zu jener fortschreitet, bei der ein theilweises Lesen möglich ist. Das Resultat kann im ersteren Falle gïnstiger ausfallen als im letzteren. So war beispielsweise ein von mir ge- 
prüftes Individuum anfänglich ansser Stande, selbst bei angestrengtem Fixiren, mit dem linken Auge Jaeger Nr. 20, 21 und 22 zu lesen; als ich zu Nr. 23 und 24 ibergegangen war und bemerkte, dass die betreffenden Worte mit Leichtigkeit gelesen wurden, kehrte ich unmittelbar darnach auf Nr. 22 zurück und fand, dass auch diese Nr., sowie die beiden vorangebenden Nr. 21 und 20 nunmehr ohne Anstrengung gelesen werden konnten.

Als Grund für diese Erscheinung lässt sich wohl annehmen, dass bei dem einmal erregten Gesichtssinn auch durch schwächere Reize Sehempfindungen bervorgerufen werden können, die vorher für sich allein die Sehfunktion nicht auszulösen vermochten ${ }^{1}$.

Die Sehprïfungen wurden mit wenigen Ausnahmen an jedem Auge separat vorgenommen und dabei eine seitliche Drehung des Kopfes, sowie ein Zusammenkneifen der Augenlider, ferner, aus später anzugebenden Gründen, auch ein Druck auf das von der Prüfung ausgeschlossene Auge sorgfältig vermieden. Die Mehrzahl der Versuchsindividuen, welche anfänglich von einer bestimmten Schriftskala nicht ein Wort wahrnahmen, dagegen bei längerem Fixiren der betreffenden Skala, diese vollständig oder theilweise zu lesen vermochten, war eine Prüfungszeit von einer Minute und etwas dariiber eingeräumt; nur bei einzelnen Personen, bei denen sich das Sehvermögen am Beginne der Untersuchung am stärksten zeigte und bei angestrengterem Fixiren rasch abnahm, wurde die am Beginne der Sehprobe vorhandene Sehfunktion als Prüfungsresultat verzeichnet.

Die an 25 Ohrenkranken in der angegebenen Weise angestellten Untersuchungen ergaben nachstehendes Resultat.

1) Vgl. Fechner, Psychophysik, Bd. 2, p. 143 und an verschiedenen anderen Stellen des genannten Werkes. Die gleiche Erscheinung findet hinsichtlich der Hördistanz statt, die sich weiter zeigt, wenn die Schallquelle aus dem Hörbereich langsam an die Hörgrenze gerückt wird, als wenn sich der Hörmesser von einem ausserhalb der Perzeptionsgrenze gelegenen Punkte dem Ohre nähert (s. Politzer, Lehrb. d. Ohrenheilk., p. 192). Wie ich mich bei Sprachübungen oftmals uiberzeugt habe, wird bei gleicher Intensität der Stimme und gleicher Entfernung des Sprechenden vom Versuchsindividuum ein bestimmtes Wort, welches die Versuchsperson anfänglich nicht zu hören vermag, von dieser ganz deutlich vernommen, wenn bei der Hörprüfung diesem Worte andere, leichter verständliche Worte vorausgeschickt werden. Vgl. ferner dies. Archiv, 1881, Bd. XXV, p. 223-342: „Ueber das An- und Abklingen acustischer Empfindungen". 


\section{Erklärung der Bezeichnungen.}

Der Wortlaut der Jaeger'schen Schriftskalen, welche bei den hier mitgetheilten Sehprüfungen zur Verwendung kamen, ist nachfolgender:

Nr. 9. "Wissenschaften entfernen sich im Ganzen immer vom Leben, und kehren nur durch einen Umweg wieder dahin zurück."

Nr. 10. "Allein kann der Mensch nicht bestehen, daher schlägt er sich gerne zu einer Parthei, weil er da, wenn nicht Rule, doch Beruhigung und Sicherheit findet."

Nr. 11. "Von Natur besitzen wir keinen Fehler, der nicht zur Tugend, keine Tugend, die nicht zum Fehler werden könnte. Diese letzteren sind gerade die bedenklichsten."

Nr. 12. „Säen ist nicht so beschwerlich als ernten. Zum Entdecken gehört Glück, zum Erfinden Geist und beide können beides nicht entbehren."

Nr. 13. "Hypothesen sind Wiegenlieder, womit der Lehrer seine Schüler einlullt.:

Nx. 14. "Der allein ist glücklich und gross, der weder zu herrschen noch zu gehorchen braucht, um etwas zu sein."

Nr. 15. „Je höher man steht, desto mehr Menschen hat man zu sehen und zu regiren, folglich desto mehr Schuldige."

Nr. 16. "Einer neuen Wahrheit ist nichts schädlicher als ein alter Trrthum."

Nr. 17. "Liebe verschenkt, Egoismus leiht."

Nr. 18. "Deutschland."

Nr. 19. "Hamburg."

Nr. 20. "Bremen。"

Nr. 21. "Danzig."

$\mathrm{Nr}$. 22. „Bern."

Nr. 23. "wem."

Nr. 24. "von."

Jeder einzelne Buchstabe, der von dem jedesmaligen Versuchsindividuum gelesen werden konnte, ist im Nachstehenden angeführt. Finden sich am Anfange oder Ende eines von der Versuchsperson gelesenen Satzes, bezw. Wortes, Punkte ver, oder sind solche zwischen den Buchstaben eingeschaltet, so soll damit angedentet werden, dass an der bezeichneten Stelle in der betreffenden Jaeger'schen Schriftskala Buchstaben oder Worte stehen, welche das Versuchsindividuum nicht zu lesen im Stande war. 
Ueber d. Einfluss von Trigeminus.Reizen auf d. Sinnesempfindungen etc. 133

Das den einzelnen Versuchen beigefügte Datum bezieht sich auf den Prüfungstag. Sämmtliche Versuche wurden in den Sommermonaten des laufenden Jahres (1882) angestellt.

R. A. $=$ Sehfunktion am rechten Auge.

L. A. = Sehfunktion am linken Auge.

$0==$ Von der betreffenden Nr. der Jaeger'schen Schriftskala kann das Versuchsindividuum keinen einzigen Buchstaben lesen.

Alles $=$ Die betreffende $\mathrm{Nr}$, der Jaeger'schen Schriftskala vermag die Versuchsperson vollständig zu lesen.

\section{Versuch I. Berger Ign, 52 Jahre.}

Rechts: Vernarbtes Trommelfell, Gehörweite für die Uhr $30 \mathrm{~cm}$ (150 cm für ein normales Ohr).

Links: Eitrige Entzündung der Paukenhöhle mit Perforation des Trommelfelles; Dauer der Affection 25 Jahre.

10.8. R. A. Jaeger Nr. $17=$ Alles; Nr. $16=0$.

L. A. Nr. $17=$ Alles, jedoch erst nach angestrengtem Fixiren; Nr. $16=0$.

23. 8. R. A. Nr. $16=$ Einer...

L. A. Nr. $16=0$; Nr. $17=$ wie am $10 . / 8$.

\section{Versuch II. Paula Bij, 18 Jahre.}

Rechtes Ohr normal. Links seit 6 Wochen eitrige Entzündung des Mittelohres mit consecutiver Entzündung des äusserèn Gehörganges.

9.8. R. A. Nr. $17=0$.

L. A. Nr. 17 = Liebe verschenkt, Egoismus....

11.|8. Die Entzündung am linken Ohre etwas gebessert.

R. A. Nr. $17=$ Liebe...

L. A. Nr. $17=$ Liebe verschenkt, Egoismus... .

16. 8. Fortschreitende Besserung links.

R. A. Nr. $17=$ L..b. ...s.h..kt, Eg.... h.

L. A. Nr. $17=$ Liebe verschenkt, Egoismus ..h.

19.8. Eitrige Entzündung sistirt.

R. A. Nr. $17=$ L..b. ...s.ch. .kt, Eg... l..ht.

L. A. Nr. $17=$ Alles; Nr. $16=$ Einer ... ist ... schädlicher . .

.. als ein alter Irrthum.

9.19. Vollständige Heilung links.

R. A. Nr. $17=$ Liebe verschenkt, Egoismus 1..h.;

Nr. $16=\ldots$ als $\ldots$ Irrthum.

L. A. $\mathrm{Nr} .16=$ Einer neuen Wahrheit $\ldots$ als ein $\ldots$ Irrthum. 
Versuch III. Julie Burg, 30 Jahre.

Rechtes Ohr normal; links seit 3 Jahren Ohrensausen bei nahezu normalem Gehör.

26. 17. R. A. Nr. $15=$ Je höher man ..., desto mehr Menschen hat man $\mathrm{zu}$.. und zu .. folglich desto mehr Schuldige.

L. A. Nr. 15 gleich dem rechten Auge, doch etwas deutlicher zu lesen.

Beim Lesen mit dem rechten Auge bemerkt Patientin häufige Verdunklungswellen, welche über das Gesichtsfeld ziehen; mit dem linken Auge wird diese Erscheinung nicht wabrgenommen.

5.|8. R. A. Nr. $14=$ Der ... ist glïcklich .. zu ...

L. A. Nr. 14 $=$ Der allein ist glücklich und gross, der w... zu ... zu ge....

\section{Versuch IV. Rud. Dem, 12 Jahre.}

Rechtes Ohr normal; links seit 8 Tagen acuter Catarrh der Paukenhöhle.

28.17. R. A. Nr. $12=$ Säen ist $\ldots$

L. A. Nr. $12=$ Säen ist ... (undeutlicher als mit dem rechten Auge).

31.7. Bedeutende Besserung links.

R. A. Nr. $12=$ Alles.

L. A. Nr. $12=\ldots$ ist nicht so beschwerlich als ernten.

20.8. Seit 14 Tagen beiderseits normales Gehörorgan.

R. A. Nr. $12=$ wie am 31.7 .

L. A. Nr. $12=$ wie am 31.7 .

Versuch V. Englinger Jos., 40 Jahre.

Linkes Ohr eitrige Entzündung der Paukenhöhle mit consecutiver Entzündung des äusseren Gehörganges; Dauer 8 Tage.

19. 8 : R. A. Nr. $17=$ Liebe $\ldots$.

L. A. Nr. $17=$ Liebe $\ldots$.

21.|8. Besserung links.

R. A. Nr. $17=$ Liebe versch...t.g... leiht.

L. A. Nr. $17=$ Liebe v.... .g....

23.8. Die Entzündung des äusseren Gehörganges (links) abgelaufen.

R. A. Nr. $17=$ Liebe verseh...t .g... leiht.

L. A. Nr. $17=$ Liebe versch...t .g...

30.|8. Die eitrige Entziundung der Paukenhöhle sistirt.

R. A. Nr. $17=0$.

L. A. Nr. $17=0$. 
Ueber d. Einfluss von Trigeminus-Reizen auf d. Sinnesempfindungen etc. 135

\section{Versuch VI. Sattler Barb.}

Seit 2 Monaten links starkes Sausen, Uhr nur beim Anlegen an die Ohrmuschel hörbar. Rechts Uhr $6 \mathrm{~cm}$. Es besteht beiderseits ein chronischer Mittelohreatarrh.

7.8. R. A. Nr. $22=$ Ber..

L. A. Nr. $22=$ B...

12.8. Uhr rechts $20 \mathrm{~cm}$; links $1 \mathrm{~cm}$.

R. A. $\mathrm{Nr}, 22=$. e.

L. A. Nr. $22=$.er..

21.8. Uhr rechts $26 \mathrm{~cm}$; links $2 \mathrm{~cm}$.

R. A. Nr. $22=$.er..

L. A. Nr. $22=$.er..

\section{Versuch VII. Gareis Ther.}

Rechts Entzündung des äusseren Gehörganges seit 5 Tagen.

7.j8. R. A. Nr. $21=$ D...

L. A. Nr. $16=\ldots$ Irrthum.

14.|8. Bedeutende Besserung.

R. A. Nr. $21=\mathrm{D} .$.

L. A. Nr. $16=\ldots$ Irrthum.

18. 8. Entzündung abgelaufen.

R. A. Nr. $21=$ Alles.

L. A. Nr. $16=\ldots$ Irrthum.

Versuch VIII. Giwisch Anton, 25 Jahre.

Rechts Cerumenpfropf im Gehörgange; seit 3 Wochen subjective Beschwerden.

28.8. R. A. Nr. $17=$ Liebe verschenkt ... lei.t.

L. A. Nr. $20=$ Alles; Nr. $19=0$.

30.|8. Der Cerumenpfropf ist seit 2 Tagen entfernt.

R. A. Nr. $17=$ Alles; Nr. $16=0$.

L. A. Nr. $19=$ Alles; Nr. $18=0$.

2.|9. R. A. Nr. $16=$ Einer $\ldots$ als ein aiter $\ldots$.

L. A. Nr. $18=$ Alles; Nr. $17=$ Liebe $\ldots$.

Versuch IX. Gugl,, 15 Jahre.

Links seit 2 Tagen eitrige Entzündung der Paukenhöhle.

26.7. R. A. Nr. $9=$ W... e... s... i... G...

L. A. Nr. $9=0$.

29.17. Links Otorrhoe sistirt. 
R. A. Nr. $9=$ Wissenschaften e... sich im Ganzen immer vom Leben und ... nur durch einen U... wieder ... zurück.

L. A. Nr. $9=$ Wissenschaften e... sich im Ganzen immer vom Leben und ... nur ...

2.18. R. A. Nr. $9=$ Wissenschaften e... sich im Ganzen immer vom Leben und ... nur durch einen U... wieder da.. zurück. Nr. $8=$ Die $\ldots$.

L. A. Nr. $9=$ Wissenschaften e... sich im Ganzen immer vom ... und ... nur durch einen $\mathrm{U}$... da.. zurück. $\mathrm{Nr} .8=\mathrm{D} \ldots$

Die am 14.|8. und 7. 9 . vorgenommenen Sehprüfungen ergaben das Resultat vom $2 . \mid 8$.

Versuch X. Janusch Friedr., 16 Jahre.

Links seit 4 Wochen eitrige Entzündung der Paukenhöhle, seit 14 Tagen Schmerz im Ohr.

10.|8. R. A. Nr. $16=$ Ein.. neuen Wahrheit ist nichts schädlicher als ein alter Irrthum. $\mathrm{Nr}^{\mathrm{r}} \mathbf{1 5}=\mathrm{J}$. .. desto mehr Menschen ....

L. A. Nr. $16=$ Ei. . : Wahrheit ist nichts ... als ein alter Irrthum. Nr. $15=$ J. .. Menschen ....

14.|8. Der Sohmerz im linken Ohre ist geschwunden.

R. A. Nr. $15=$ J. .. desto mehr Menschen ... zu ... folglich desto mehr Schuldige.

L. A. Nr. $15=\ldots$ desto ... Menschen ... folglich desto mehr Schuldige.

16.|8. Links Otorrboe geringer.

R. A. Nr. $15=J$. ... desto mehr Menschen ... zu ... und ... folglich desto mehr Schuldige.

L. A. Nr. $15=$ J. ... desto mehr Menschen ... zu ... folglich desto mehr Schuldige.

Versuch XI. Koch Jac., 24 Jahre.

Links seit 10 Wochen eitrige Entzündung der Paukenhöhle.

12.8. R. A. Nr. $18=$ De.t...land (undeutlich).

L. A. Nr. $18=$ De.t. . .land (deutlich).

14.|8. Links Besserung.

Nr. 18 wird mit dem rechten und linken Auge gleich deutlich gelesen.

R. A. Nr. $17=$ Liebe .er..henkt .g.i.m.s ...

L. A. Nr. $17=$ L. . be .e...henkt ...

Versuch XII. Landgraf Jos., 11 Jahre.

Rechts seit 6 Jahren Schwerhörigkeit, seit 1 Jahre PaukenhöhlenPolyp. 
Ueber d. Einfluss von Trigeminus-Reizen auf d. Sinnesempfindungen etc. 137

10.|8. R. A. Nr. $15=\mathrm{J}$. .

L. A. Nr. $15=\mathrm{J} .$.

14.|8. R. A. $\mathrm{Nr} .15=\mathrm{Je} \ldots$ desto mehr ... hat ... folglich desto mehr Schuldige.

L. A. Nr. $15=J e \ldots$ desto mehr Schuldige.

17.j8. R. A. Nr. $15=J_{e} \ldots$ man steht desto mehr Menschen hat man $\mathrm{zu}$... folglich desto mehr Sohuldige.

L. A. Nr. $15=$ Je ... man steht, desto mehr ... hat man zu ... folglich desto mehr Schuldige.

\section{Versuch XIII. Lemberger, 14 Jahre.}

Bilateral chron. Mittelohrcatarrh; seit 8 Tagen nach einem Sturze erhöhte Schwerhörigkeit bei nachweislicher Hyperämie der Tromelfellgefässe. Patient ist rechts amblyopisch und wird daher nur am linken Auge geprüft.

26.7. Nr. $11=$ Von Natur besitzen wir keinen Fehler, der nicht zu....

31.7. Besserung der Schwerhörigkeit.

Nr. $11=$ Alles; Nr. $10=$ Alles;

Nr. $9=\ldots$ sich im G... immer vom Leben und .... durch einen Umweg wieder .....

24. 8. Fortschreitende Besserung.

Nr. $9=\ldots$ sich $\ldots$ immer $\ldots$ Leben .. und $\ldots$

28.|8. Nr. $9=$ Wissen..... sich im Gan... immer ... Leben und .... durch einen Umweg wieder dahin zurück.

Versuch XIV. Lor., 12 Jahre.

Rechts eitrige Entzündung der Paukenhöhle, seit 1 Jahre wiederholt rïckfällig.

27.|7. R. A. Nr. $12=$ Säen $\ldots$.

L. A. Nr. $12=$ Säen ist $\ldots$.

5.|8. R. A. Nr. $12=$ Säen ist nicht so beschwerlich als ernten. Zum Entdecken gehört .... Nr. $11=$ Von Natur b....

L. A. Nr. $11=$ Von Natur .... wir keinen Fehler ....

\section{Versuch XV. Nepach, 54 Jahre.}

Links seit 14 Tagen eitrige Entzündung der Paukenhöhle. Patient bemerkt seit einigen Tagen eine auffallige Herabsetzung seiner Sehfunktion.

8.18. R. A. Nr. $18=$ Alles; Nr. $17=0$.

L. A. Nr. $15=$ Je höher $\operatorname{man} \ldots \ldots$.

11.j8. Besserung.

R. A. $\mathrm{Nr} .18=$ Alles; Nr. $17=0$.

L. A. Nr. $15=$ Je höher man ... desto mehr Menschen hat man zu ... und zu regieren, folglich d.... 
16.|8. Otorrhoe (links) sistirt.

R. A. $\mathrm{Nr} .17=$ Liebe verschenkt, Egoismus ....

Nr. $16=$ Eine. neue. Wahrheit .... alter Irrthum.

L. A. Nr. $15=$ Alles;

Nr. $14=$ Der ... ist glücklich und $\mathrm{g} .$. der ....

28.8. L. A. Nr. $14=$ Der $\ldots$ ist glücklich und gross, der $\ldots$ herrschen ... zu gehorohen braucht, um ....

\section{Versuch XVI. Ortner Kar., 21 Jahre.}

Seit 1 Jahre links eitrige Entzïndung der Paukenhöhle, seit 5 Tagen Entzündung des äusseren Gehörganges.

R. A. Nr. $15=$ Je ... $\operatorname{man} \ldots$ desto $\operatorname{mehr} \ldots$ ha. ... und ...

L. A. Nr. $15=$ Je ... man ... desto mehr Menschen ....

26. $\mid 8$. R. A. Nr. $15=$ Je ... $\operatorname{man} \ldots$ desto $\ldots$ Menschen $\ldots$. Nr. $14=\ldots$ ist $\ldots$

L. A. Nr. $15=$ Alles; Nr. $14=$ Der . . . ist ... und ... der ... zu ...

20.|9. R. A. Nr. $14=\mathrm{D} . . \ldots$ ist $\ldots$ and $\ldots$ der $\ldots$ zu $\ldots$

L. A. Nr. $14=$ Der ... ist ... und ... zu ... noch $\mathrm{zu} \ldots$

Versuch XVII. Peras Anna, 17 Jahre.

Seit 5 Tagen nach einem Schlage auf das linke Ohr, Otalgia tympanica.

16.|8. R. A. Nr. $17=$ Liebe verschenkt ... le..t.

L. A. Nr. $17=$ Liebe ....

19.|8. Otalgie sehr unbedeutend.

R. A. $\mathrm{Nr} .17=$ Liebe verschenkt, Egoismus le..t.

L. A. Nr. $17=$ Liebe verschenkt, Egoismus le..t.

Versuch XVIII. Pren Karl, 50 Jahre.

Seit 40 Jahren eitrige Entzïndung der Paukenhöhle links; Polyp daselbst.

9.|8. R. A. Nr. $17=$ Alles; Nr. $16=$ Einer neuen W... ist nichts schädlicher als ein alter Irrthum.

L. A. $\mathrm{Nr}, 17=$ Liebe versch...., Nr. $16=0$.

16.|8. Seit Entfernung des Polypen aus dem linken $\mathrm{Ohr}$ ist die früher profuse Otorrhoe sehr gering.

R. A. Nr. $16=$ Alles; Nr. $15=$ J. höh. ... Menschen .. zu ..

L. A. Nr. $17=$ Alles; Nr. $16=\ldots$ ist nicht . . ein ...

23.|8. Otorrhoe links nur spurweise.

R. A. Nr. $15=$ J. h... steht ... Menschen ...

L. A. Nr. $16=$ Einer neuen $\ldots$. ist nichts schädlicher als ein alter Irrthum. $\mathrm{Nr} .15=\mathrm{J} . \ldots \ldots$ 
Ueber d. Einfluss von Trigeminus-Reizen auf d. Sinnesempfindungen etc. 139

30./8. Otorrhoe sistirt.

R. A. Nr. $15=$ J. ... mehr Menschen ... zu $\ldots$

L. A. $\mathrm{Nr} .15=\mathrm{J} . \ldots$ Mensehen ...

20./9. R. A. Nr. $15=$ J. ... Menschen ...

L. A. Nr. $15 \mathrm{~J} . \ldots \mathrm{h} . \ldots$.

Versuch XIX. Schafer Franziska.

Schwerhörigkeit bilateral seit Oktober 1881. Beiderseits Cerumenpfröpfe im äusseren Gehörgange.

31./8. R. A. Nr. $17=$ Liebe $\ldots$.

L. A. Nr. $17=$ Liebe ....

2./9. Seit der Ausspritzung beider Cerumenpfröpfe (am 31./8.) beiderseits gutes Gehör.

R. A. Nr. $17=$ Liebe ...sch...

L. A. $\mathrm{Nr} \cdot 17=$ Liebe $\ldots$.

9./9. R. A. Nr. $17=$ Liebe verschenkt, Ego. .mus leiht.

L. A. Nr. $17=$ Liebe verschenkt, Ego..mus leiht.

Versuch XX. Schafer Rudolf, 14 Jahre.

Rechts seit 6 Monaten eitriger Ausfiuss aus dem Ohre; Polyp der Paukenhöhle.

8./8. R. A. Nr. $24=0$.

L. A. Nr. $18=$ Alles; Nr. $17=0$.

12./8. R. A. Nr. $24=0$.

L. A. Nr. $17=$ Liebe $\ldots$

17./8. R. A. Nr. 24, 23, 22 und $21=$ Alles.

L. A. Nr. $17=$ Liebe ...

19./8. Fortschreitende Besserung.

R. A. Nr. $21=$ Alles.

L. A. Nr. $17=$ Liebe verschenkt $\ldots$

29./8. R. A. $\mathrm{Nr}, 21=$ Alles.

L. A. Nr. $17=$ Liebe $\ldots$

Versuch XXI. Schibek Jul., 16 Jahre.

Rechts seit 6 Monaten Schwerhörigkeit; Mittelohrcatarrh; Uhr $6 \mathrm{~cm}$. Links normal.

7./8. R. A. Nr. $14=$ Der allein ist glücklich und gross, der weder $2 u$ ... zu ...

L. A. Nr. 16 = Einer neuen W....

9./8. R. A. Nr. 14 wie am 7./8.

L. A. Nr. $16=$ Einer neuen Wahr.... 
11./8. Uhr rechts $8 \mathrm{~cm}$.

R. A. Nr. 14 wie am $7 . / 8$.

L. A. Nr. $16=$ Einer neuen Wahrheit .....

16./8. Uhr rechts $12 \mathrm{~cm}$.

R. A. Nr. $14=$ Der allein $\ldots$ glücklich $\ldots$.. der weder zu herrschen noch zu ... braucht, um ... zu sein.

Nr. $13=H .$. sind $W$.. wo..t der L.. ... Sch...

L. A. Nr. $16=$ Einer neuen Wahrheit ist nichts $\ldots$ als ein alter Irrthum.

31./8. Rechts Uhr $25 \mathrm{~cm}$; am rechten Ohreingange ein kleiner Abscess.

R. A. Nr. $14=$ Alles; Nr. $13=$ H... sind W...l... wo..t der L.h. . ... Sch.1...

L. A. Nr. $15=$ Je höher man steht, d.st. mehr Menschen hat man zu ... folg... desto mehr Schuld....

4./9. Uhr rechts $34 \mathrm{~cm}$.

R. A. Nr. $13=$ H...sen sind W...l... womit der L.h... ..i.. Schüler ....

L. A. Nr. $15=$ Je höher man st..t, desto m... M. .sch.. hat man zu s.h.. .. desto mehr Sch.1...

\section{Versuch XXII. H. Schmid.}

Trommelfell am linken Ohre rupturirt.

9.7. R. A. Nr. $10=0$.

L. A. Nr. $10=0$.

Das Gesichtsfeld erscheint dem linken Auge heller als dem rechten.

31.17. Nr. 10 wird weder mit dem rechten noch dem linken Auge gesehen.

8.18. Linkes Trommelfell erscheint vollständig geheilt.

R. A. Nr. $10=$ Allein .... Mensch ... daher schlägt ...

L. A. Nr. $10=$ Allein kann der Mensch nicht ..... daher schlägt er sich gerne $\mathrm{zu} . .$.

Versuch XXIII. Dr. Uyh.

Beiderseits hochgradiger chronischer Mittelohrcatarrh. Rechtes Auge seit 20 Jahren post Trauma sehwachsichtig. Täglich findet eine Bougirung beider Tubenkanäle statt.

16./8. R. A. Nr. $24=0$.

L. A. $\mathrm{Nr} 13=\mathrm{H} . \ldots$ sind ...

17./8. L. A. Nr. $13=$ H.... sind Wiegenlieder, womit der Lehrer seine Schüler einlullt;

Nr. $12=\ldots$ ist nicht so b... als Zu... zum ...

18./8. L. A. Nr. 13 wie am 17./8.; Nr. $12=\ldots$ ist nicht so be... als .... Zum ... g... Glück, zum .... 
Ueber d. Einfluss von Trigeminus-Reizen auf d. Sinnesempfindungen etc. 141

25./8. Patient beobachtet seit der Ohrenbehandlung eine auffällige Sehbesserung am rechten Auge, ferner an beiden Augen eine merkliche Aufhellung des Gesichtsfeldes. Während Patient bisher nur mit Mühe lesen oder schreiben konnte, da binnen wenigen Minuten die Buchstaben undeutlich, wie verschwommen erschienen, besteht gegenwärtig ein gebessertes und gleichmässig anhaltendes Sehvermögen.

R. A. Nr. $22=$ B.rn; Nr. $21=$ D...

L. A. wie am $17 . / 8$.

Versuch XXIV. Dr. Walln.

Links seit 9 Monaten eitrige Entzündung der Paukenhöhle.

26.77. R. A. Nr. $17=$ Lie....

L. A. $\mathrm{Nr} \cdot 17=$ Liebe $\ldots$.

29./7. R. A. Nr. $17=0$.

L. A. Nr. $17=$ Liebe ... sch....

2/8. Links Entzündung des äusseren Gehörganges.

R. A. Nr. $17=0$.

L. A. Nr. $17=$ Lie....

14./8. Die Entzündung des Gehörganges abgelaufen; Otorrhoe links sistirt.

R. A. Nr. $17=0$.

L. A. Nr. $17=0$.

24./8. R. A. Nr. $17=0$.

L. A. Nr. $17=$ Liebe ......

6./9. R. A. Nr. $17=0$.

L. A. Nr. $17=$ Liebe .....

Versuch XXV. Stögermeyer.

Rechts Entzündung des äusseren Gehörganges.

2./8. R. A. Nr. $14=0$.

L. A. Nr. $14=$ Der allein ist glücklich und gross, der ...

4./8. Besserung.

R. A. Nr. $14=$ Der ...

L. A. Nr. $14=\mathrm{D}$. . . .

7.j8. Die Ohrenentzündung ist vollständig zurückgegangen.

R. A. Nr. $14=$ Alles.

L. A. Nr. $14=0$.

Wie aus den mitgetheilten Fällen ersichtlich ist, war unter 25 Ohrenkranken, an Seite des erkrankten bez. stärker affizirten Ohres, das Sehvermögen im Vergleiche mit dem des anderen Auges 11 mal (Fall I, IV, VI, VII, IX, X, XIV, XVII, XVIII, 
$\mathrm{XX}, \mathrm{XXV}$ ) herabgesetzt, $8 \mathrm{mal}$ (II, III, VIII, XI, XV, XXI, XXII, XXIV) erhöht und 4 mal (V, XII, XVI, XIX) gleich; bei zwei Versuchspersonen (XIII, XXIII) kam überhanpt nur 1 Auge zur Prüfung.

Wenngleich das hier angeführte Zahlenverhältniss anscheinend gegen einen Einfluss der Erkrankungen des äusseren oder mittleren Ohres auf das Sehvermögen spricht, so muss doch dagegen der Einwand erhoben werden, dass demselben überhaupt keine Bedeutung beigelegt werden kann, da ja in den betreffenden Fällen die Sehfunktion vor dem Beginne der Ohrenaffektion nicht bekannt war und also eine durch die Ohrenerkrankung möglicherweise eingetretene Veränderung des Sehvermögens sich jeder Beurtheilung entzieht.

Um sich also hiertiber Aufschluss zu verschaffen, musste man zu erfahren suchen, ob bei den einzelnen Ohrenpatienten, die Veränderungen in der Erkrankung des äusseren oder mittleren Ohres, auch von einer Veränderung des Sehvermögens begleitet waren. Aus diesem Grunde habe ich bei jedem Versuchsindividuum in den verschiedenen Stadien der Ohrenaffektion wiederholte Sehprüfungen angestellt, deren Resultate oben verzeichnet sind. Denselben ist folgendes zu entnehmen:

Unter 25 Fällen zeigte sich das Sebvermögen, während der Abnahme des Ohrenleidens, $21 \mathrm{mal}$ gebessert u. z. fand in $2 \mathrm{Fällen}$ (I, VII) eine ganz unwesentliche Besserung statt, indem am Schlusse der Behandlung nur einzelne Buchstaben oder Silben der betreffenden Schriftskala mehr gelesen werden konnten, als im Beginne der Behandlung, 5 mal (III, IV, XVI, XVII, XIX) erstreckte sich die Sehbesserung auf mehrere Worte der Schriftskala und 14 mal hob sich das Sehvermögen um 1-4 Nr. der Jaeger'schen SchriftVorlagen. Unter den zuletzt erwähnten 14 Fällen vermochten 4 Versuchsindividuen (IX, X, XII, XXII) am Beginne der Behandlung nur einzelne Buchstaben oder Silben einer bestimmten Nr., am Ende der Behandlung dagegen alles oder wenigstens nahezu alles zu lesen; bei 4 Patienten (II, XI, XIV, XXI) stieg das Sehvermögen etwas ïber $1 \mathrm{Nr}$., bei 3 (XIII, $\mathrm{XV}, \mathrm{XVIII)}$ ) um $2 \mathrm{Nr}$., bei 2 (VIII, XXIII) um $3 \mathrm{Nr}$., in 1 Falle (XX) um 4 Nr. In den übrigen 4 Fällen zeigte sich das Sehvermögen bei einem Patienten (XXIV) trotz der Besserung des Ohrenleidens unverändert, bei einem anderen (VI) als nahezu unverändert und bei einem 3. (V) sogar als verschlimmert. Der 4. Fall (XXV) nimmt unter den 25 
Fällen insofern eine Ausnahmsstellung ein, als anfänglich bei diesem, an Seite des erkrankten rechten Ohres, ein auffällig geringes Sehvermögen bestand, gegenüber dem des anderen Auges, während im Verlaufe der Besserung der Ohrenentztindung und besonders nach Ablauf derselben, ein Sinken der Sehkraft am Auge der gesunden Seite und ein Steigen derselben am anderen Auge eintrat, so zwar, dass Jaeger Nr. 14 im Beginne der Entzündung des rechten Ohres mit dem linken Auge theilweise, mit dem rechten Auge gar nicht, nach Ablauf der Entzündung dagegen mit dem rechten Auge vollständig gelesen werden konnte, indess das linke Auge nunmehr nicht einen Buchstaben wahrzunehmen vermochte.

Wenn man von den oben erwähnten 21 Fällen mit Sehbesserung die angefiubrten 2 Fälle mit unbedeutender Sehzunahme und die weiteren 5 Fälle mit mässiger Steigerung der Sehfunktion abrechnet und für diese Fälle die Möglichkeit zulässt, dass bei ihnen die Sehbesserung nicht infolge des gebesserten Ohrenleidens eingetreten sei, sóndern auf Schwankungen der Sehfunktion beruhe, die von der Ohrenaffektion vollständig unabhängig sein können, so erübrigen noch immer 14 Fälle (unter 25 Fällen), in denen ein Zusammenhang zwischen Ohrenerkrankung und Sehvermögen nicht von der Hand zu weisen ist.

Es lässt sich demnach auf Grundlage der mitgetheilten Versuchsresultate wohl mit ziemlicher Sicherheit behaupten, dass eine Erkrankung des äusseren oder mittleren Ohres einen Einfluss auf das Sehvermögen nehmen kann, und dass ein solcher Einfluss im Allgemeinen sehr häufig and in einzelnen, keineswegs seltenen Fällen sogar als erheblich erscheint.

Erwähnenswerth ist der Umstand, dass die hauptsächlichste Hebung der Sehkraft unter den beobachteten 25 Fällen häufig innerhalb die ersten Behandlungstage fiel und sich im Verlaufe der weiteren Besserung des Ohrenleidens ungefähr auf derselben Höhe erhielt. Diese Erscheinung war auch bei zweien der von mir Eingangs erwähnten Patienten eingetreten.

Ein andermal gibt sich dagegen eine langsam zunehmende Sehbesserung zu erkennen, welche zu der allmählichen Besserung des Obrenleidens in einem Abhängigkeitsverbältniss steht, oder aber das Sehvermögen nimmt bei gleichbleibendem Zustande des Hörorgans, bez. nach vollständiger Heilung des Ohrenleidens, noch 
durch einige Zeit stetig $z u$, in einzelnen Fällen dagegen wieder um etwas ab.

Die Versuche ergaben ferner die interessante Thatsache, dass ein einseitiges Ohrenleiden nicht nur das gleichseitige Auge, sondern auch das Auge der anderen Seite zu beeinflussen vermag, wenigstens trat in der Mehrzahl der Fälle von einseitiger Ohrenerkrankung auch am entgegengesetzten Auge eine Sehbesserung: ein, ja diese erschien zuweilen daselbst viel beträchtlicher als am anderen, dem erkrankten Ohre entsprechenden Auge. So hob sich das Sehvermögen im Falle VIII, nach der Ausspritzung eines Cerumenpfropfens aus dem rechten Gehörgange, am rechten Auge von Jaeger Nr. 17 auf Nr.16, links jedoch von Nr. 20 auf Nr. 17. In anderen Fällen wieder nimmt mit der Besserung einer einseitigen Ohrenaffektion das Sehvermögen auf dem gleichseitigen Auge bedeutender za als am entgegengesetzten Auge und kann dieses vorher vielleicht bessere Auge schliesslich sogar übertreffen; ein andermal wieder steigt das Sehvermögen an dem anfänglich schlechter perzipirenden Auge rascher an als am anderen, so dass schliesslich beide Angen die gleiche Sehkraft aufweisen (Fall XVIII). Viel seltener scheinen Fälle vorzukommen wie Fall XXV, in denen während der Abnahme einer einseitigen Ohrenentzindung eine Verschlimmerung des Sehvermögens am entgegengesetzten Auge, bei gleichzeitiger Besserung der Sehfunktion am Auge der erkrankten Seite erfolgt. Derartige Fälle könnten in dem Sinne gedentet werden, dass eine einseitige Ohrenerkrankung am Auge der entsprechenden Seite das Sehvermögen herabzusetzen und dabei gleichzeitig die Sehkraft am anderen Ange zu steigern vermöge und dass beim Nachlass der Ohrenaffektion, mit der Abnahme der auf einer Irritation bewirkten Sehbesserung am letzteren Auge, gleichzeitig eine Zunahme des durch das Ohrenleiden verminderten Sehvermögens am ersteren Auge zu. Stande kommt.

Da die besprochenen Versuchsergebnisse die Möglichkeit einer Beeinflussung des Sehvermögens durch pathologische Vorgänge im äusseren oder mittleren Ohre erwiesen hatten, so versuchte ich nunmehr durch weitere Untersuchungen zu erfahren, ob auch die einzelne Reizeinwirkung auf das $\mathrm{Ohr}$ für das Sehvermögen von irgend welcher Bedeutung sei. Ich nahm zu diesem Zwecke in einer Reihe von Fällen unmittelbar vor und nach einer Einwirkung auf das Obr Sehprïfungen vor und theile im Folgenden einige Beispiele mit: 
Ueber d. Einfluss von Trigeminus-Reizen auf d. Sinnesempfindungen etc. 145

1) Gugl. R. A. Jaeger Nr. $9=W \ldots$ e... s... i.. G... L. A. $"$ Nr. $9=0$.

Nach einer Lufteinblasung in beiden Ohren tritt zuerst am rechten und 8 Sekunden später auch am linken Auge eine Aufhellung des Gesichtsfeldes ein.

R. A. Nr. $9=$ Wissenschaften e... sich im Ganzen immer vom ...

L. A. Nr. $9=$ Wissenschaften e... sich im Ganzen ....

2 Minuten später erscheint die Sehkraft auf beiden Augen ganz gleich; nach 3 Minuten sinkt das Sehvermögen anfangs am linken, dam auch am rechten Auge bis auf die frühere Stufe zurück. An einem andern Tage erregt die Luftdouche eine Ferschlimmerung des Sehvermögens, die nach 4 Minuten schwindet.

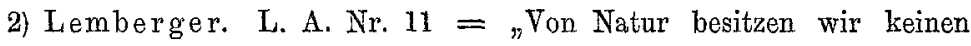
Fehler, der nicht zur" ${ }^{*}$.

Nach einer Lufteinblasung in das Mittelohr liest die Versuchsperson nunmehr auch die folgenden Worte: ...Th Tugend, keine Tugend, die nicht zum Fehler werden $\mathrm{k}$ "....

3) H. Leys. Jaeger Nr. $13=\ldots$, sind Wiegenlieder, womit der Lehrer seine Schüler" ....

Nach Bougirung der rechten Ohrtrompete wird von Nr. 13 Alles gelesen, ferner von $\mathrm{Nr} .12=\ldots$, ist nicht so beschwerlich"...

4) Ondriska, R. A. und L. A. Nr. $17=$ „Liebe“...

Nach einer Lufteinblasung in beide Ohren mittelst des Politzer'schen Verfahrens, vermögen sowohl das rechte als auch das linke Auge $\mathrm{Nr} .17$ vollständig zu lesen; 7 Minuten später jedoch wieder nur das erste Wort: "Liebe."

5) Pechhan. Von Nr. 20 werden nur Striche, jedoch keine Buchstaben ausgenommen. Nach einer Luftdouche in beide Ohren erscheint unmittelbar darauf der Buchstabe "B“, 30 Sekunden später plötzlich das ganze Wort "Bremen"; 1 Minute später nimmt das Sehvermögen etwas ab, nach 2 Min. zeigt sich das Wort sehr undeutlich, 1 Minute später dagegen wieder deutlicher, nach einer weiteren Minute wieder schwach und bleibt so bis am Schlusse der durch 15 Minuten fortgesetzten Prüfung unverändert.

Es findet nunmehr die Prüfung mit Jaeger Nr. 18 statt, von dem die Versuchsperson nur den ersten Buchstaben "D* zu lesen vermag. Nach einer Lufteinblasung in beide Ohren tritt 10 Sekunden später das Wort „Deutschland" plötzlich deutlich hervor; nach 2 Minuten erscheint die erste Silbe "Deutsch" heller als die 2. Silbe "land", eine Minute später zeigt sich das ganze Wort gleich hell; nach 5 Minuten wird jedoch nunmehr "De.....d" gelesen und einige Sekunden darauf nur "D*.

Im Verlaufe der weiteren Prüfung gibt sich keine Aenderung des Sehvermögens zu erkennen. Bei einem 2. Versuche tritt nach einer Luftdouche 
der Ohren abermals das ganze Wort „Deutschland“ deutlich hervor, doch 5 Sekunden später wird nur „D“ gesehen.

6) H. Reckensch.

R. A. Nr. 14 mit grosser Anstrengung: "Der allein ist glïcklich und gross".....

L. A. Nr. $14=$ Alles.

In den rechten Tubenkanal wird eine Bougie bis durch den Isthmus tubae eingeführt; unmittelbar darauf liest das rechte Auge von Nr. $14=$ "Der allein ist glücklich und gross, der weder zu herrschen" .......; eine Minute später auch die folgenden Worte: „noch zu ge... um etwas zu sein". Dem linken Auge erscheint die Schrift deatlicher als vorher. Nach 5 Min. geht das Sehvermögen wieder zurück.

'H. Schm. vermag von Jaeger Nr. 10 nur den ersten Buchstaben "A $A^{*}$ zu lesen. Nach der Einführung eines Tampons durch die vorhandene Trommelfell-Lücke in die Paukenhöhle liest Patient: "All... kann der Mensch".... Nach 2 Minuten schwindet die Sehbesserung und wiederholt sich abermals anf einige Sekunden nach einer Lufteinblasung ins Ohr. Es wird nunmehr gepulverte Borsäure in die Paukenhöhle eingeblasen; gleich darauf nimmt Patient von Nr. 10 die Worte: „All... kann der Mensch nicht be"... dentlich aus, doch bereits nach wenigen Sekunden nur den ersten Buchstaben "A".

Bei demseiben Patienten finden an einem andern Tage weitere Versuche statt. Von Nr. 10 erscheint abermals nur "A". Nach Entfernung eines im Gehörgange seit einigen Tagen gelegenen Tampons liest Patient plötzlich deutlich: „All... kann der Mensch nicht be... daher schlägt er sich".... 2 Minuten später ist die Sehbesserung zurückgegangen. Nach einer Ausspritzung der mit eitrigem Sekrete theilweise erfüllten Pankenhöhle, erfolgt abermals eine Steigerung des Sehvermögens, und Patient liest: „All... kann der Mensch nicht be...., daher schlägt? er sich gerne"..... Die Besserung währt nur durch einigé Sekunden.

8) Stieglitz. Jaeger Nr. 22 wird mit einem rothen Seidenpapier bedeckt, durch welches der Untersuchte keinen Buchstaben von dem Worte "Bern" ausnimmt. Nach einer Lufteinblasung ins Ohr treten plötzlich die Buchstaben "er" deutlich hervor, 10 Sekunden später auch "B", also "Ber". Die nach 2 Minuten eintretende Sehverschlimmerung wird durch eine abermalige Lufteinblasung rasch behoben und so auch ein zweitesmal zwei Minuten später. Ich stellte nun den Versuch an, ob das Sehvermögen durch rasch aufeinanderfolgende Lufteinblasungen in beide Ohren eine bedeutendere Steigerung erfahren könne; es ergab sich in der That, dass nach viermaliger Wiederholung des Politzer'schen Verfahrens eine bedeutende Aufhellung des Gesichtsfeldes entstand und dabei der bisher nicht lesbare Buchstabe " $\mathrm{n}^{\text {" }}$ hervortrat, demnach das ganze Wort "Bern" von dem Untersuchten wahrge- 
nommen wurde. Diese Sehbesserung hielt 15 Minuten hindurch an. Eine gleiche Hebung der Sehkraft ergab ein Inductionstrom bei Application der einen Electrode an den Ohreingang, der andern Electrode an den Hals.

9) Dr. Uyh.

L. A. Nr. $13=$ "H.... $\operatorname{sind}^{\prime \prime} \ldots$

Nach Bougirung des Tubenkanales der anderen rechten Seite, 1 Min. später: L. A. Nr. $13={ }$ H... sind .... womit der Lehrer seine Schüler ein".....

Dieselbe Sehbesserung ist auch 10 Minuten später nachzuweisen, erscheint aber am nächsten Tage verschwunden.

10) H. Weiss. Nr. $10=0$. Eine Bougirung des rechten Tabenkanales bewirkt keine Sehbesserung; bei Bougirung der linken Ohrtrompete treten die Worte ....der" und "nicht"... hervor, gehen jedoch noch während die Bougie im Tubenkanale liegt wieder zurück. Es wird nunmehr die Bougie entfernt and danach Luft in die linke Paukenhöhle eingeblasen; 30 Sekunden später erscheinen die Worte: ....,kann der .... nicht".... Nach 4 Minuten ist die Sehbesserung zurückgegangen. An einem anderen Versuchstage erfolgt bei Bougirung des rechten Tubenkanales eine rasch voräbergehende Sehsteigerung nur am rechten Auge, bei Bougirung der linken Ohrtrompete nur am linken Auge.

11) Falmeier. Links acute eitrige Entzündung der Paukenhöhle.

R. A. Nr. $15=$ Je ... man steht $\ldots$ desto mehr Menschen hat $\operatorname{man} \mathrm{zu} .$. und ...

L. A. Nr. $15=$ J....

Nach Lufteinblasung in beide Ohren, $1{ }^{1} / 2$ Minuten später:

R. A. Nr. $15=$ Je ... man ... desto mehr Menschen hat man $\mathrm{zu}$... folglich desto mehr Schuldige.

L. A. Nr. $15=$ dem rechten Auge.

Nach 1//2 Minuten geht die Sehbesserung links zurück, 2 Minuten darauf auch am rechten Auge.

12) Pschiwanek. Rechts eitrige Entzündung der Paukenhöhle.

R. A. Nr. $14=$ D.... (undeutlich).

L. A. Nr. $14=$ D.... (deutlich).

Nach einer Lufteinblasung in beide Trommelhöhlen schwindet der Buchstabe "D" sowohl dem rechten als dem linken Auge und tritt erst nach 2 Minuten wieder hervor. Wiederholte Versuche ergaben ein gleiches Resultat. Dagegen erscheint an einem anderen Versuchstage nach der Lufteinblasung in das $\mathrm{Ohr}$ eine vorübergehende Steigerung des Sehvermögens. 
Den mitgetheilten Versuchen ist zu entnehmen, dass verschiedene Reizeinwirkungen auf das äussere und mittlere Ohr zuweilen einen auffälligen Einfluss auf das Sehvermögen ausüben, der sich gewöhnlich in einer Steigerung, mitunter in einer Verminderung der Sehstärke äussert und zwar können gleiche Reize auf das Ohr bei demselben Individuum einmal eine Verbesserung, ein andermal eine Verschlimmerung der Sehfunktion bedingen (s. Fall 12). Die Besserung des Sehvermögens erfolgt entweder unmittelbar anf die vorausgegangene Einwirkung auf das Ohr oder binnen kurzer Zeit, gewöhnlich nach einigen Sekunden; sie kann jedoch auch etwas später eintreten (in dem Falle 9 nach 1 Minute).

Gewöhnlich steigt das Sehvermögrn sehr rasch an, zuweilen anscheinend plötzlich, ein andermal mehr allmählich oder sprungweise. Die Zunahme des Sehvermögens tritt bei gleicher Einwirkung auf beide Ohren nicht an beiden Augen gleich stark herror, zuweilen auf dem anfänglich schlechter perzipirenden Auge bedeutender als am ursprïnglich besseren Auge, wodureh das Sehvermögen beiderseits ein gleiches werden kann (s. Fall 1). Je nach der Intensität der Reizeinwirkung auf das Ohr bez. auf beide Ohren erweist sich der Grad der Sehsteigerung sehr verschieden, sowie auch durch Summirung der Reizeffekte eine weitere Erhöhung des Sehvermögens herbeigeführt werden kann (s. Fall 8).

In anderen Fällen dagegen schwächt sich die Wirkung mit der Wiederholung des Reizes allmählich ab oder tritt überhaupt nur bei der ersten Reizeinwirkung auf das Ohr auffällig hervor. Der das eine Ohr treffende Reiz übt seinen Einfluss auch auf das andere Auge aus, nur bei schwächerer Einwirkung erscheint in einzelnen Fällen bloss das Sehvermögen des Auges an der gereizten Seite allein beeinflusst.

Die Daner der Sehbesserung ist mitunter nur momentan und wird dann sehr leicht vollständig: thersehen; in der Mehrzahl der von mir beobachteten Fälle betrug jedoch die Dauer 1-4 Minuten und erst nach dieser Zeit sank das Sehvermögen mehr oder minder rasch auf die frühere Stufe zurïck. Die Besserung kann sich jedoch auch auf 5-10 Minuten erstrecken und hielt in einem meiner Fälle sogar mehrere Stunden an. Manchmal finden sich, nach einer Reizeinwirkung auf das $\mathrm{Ohr}$, durch einige Zeit Schwankungen der Sehstärke vor, wobei Besserungen mit Verschlimmerungen des Sehvermögens abwechseln (s. Fall 5). 
Ueber d. Einfluss von Trigeminus.Reizen auf d. Sinnesempfindungen etc. 149

Wie ich bereits Eingangs erwähnte und wie auch ans den angeführten 25 Beobachtungsfällen (s. S. 133 u. folg.) hervorgeht, kann eine einmalige Reizeinwirkung auf das $\mathrm{Ohr}$ eine anhaltende Sehbesserung erregen. Hierbei scheint die Intensität des Reizes auf die Grösse und Dauer des Effektes von grossem Einflusse zu sein, so findet z. B. bei einer Lufteinblasung in das Ohr mittelst des Politzer'schen Verfahrens, sowie beim Katheterismus der Ohrtrompete, nicht selten eine viel geringere Steigerung des Sehvermögens statt als bei der bedentend energiseher wirkenden Einfithrung einer $5 / 4-1 \frac{1}{2} \mathrm{~mm}$ dicken Bougie durch den Isthmus tubae. Die Abhängigkeit des Seheffektes von der Intensität des auf das Ohr einwirkenden Reizes änsserte sich am deutlichsten bei den von mir zuerst erwähnten Fällen, in welchen das Sehvermögen trotz zahlreicher Lufteinblasungen ins $\mathrm{Ohr}$ und Monate hindurch vorgenommener Katheterisationen des Tubenkanales keine merkliche Aenderung erfuhr, indess eine einmalige bez. zweimalige Bougirung der Ohrtrompete eine höchst beträchtliche Sehbesserung erzielte, die in dem einen Falle gegenwärtig seit einen Jahre, in dem anderen Falle seit 6 Monaten unverändert anhält.

Anmerkung. Bei meinen Versuchen betreffs des Grades der Sehsteigerung infolge von Reizung des äusseren oder mittleren Ohres fiel es mir auf, dass bei einzelnen Individuen, nach éiner Lufteintreibung ins Mittelohr, ein Ansteigen des Sehvermögens in ganz eigenthümlicher Weise erfolgt. Während die Sehbesserung nach einer Lufteinblasung gewöhnlich rasch binnen wenigen Sekunden, oder wenigstens nach einigen Minuten den höchsten Stand zu erreichen pflegt, bemerkte ich an einzelnen Versuchspersonen, nach der OhrenLuftdouche, ein stetig ansteigendes Sehvermögen, welches selbst bei einer $1 / 2-1$ stiundigen Sehprüfung noch nicht abgeschlossen erschien. Ich stellte daher zunächst an diesen, später auch an anderen Individuen, obne vorausgeschickte Lufteinblasungen ins Mittelohr, eine Reihe aufeinanderfolgender Sehprufungen an, wobei ich für jede einzelne Sehprüfung als Zeitdauer 1 Minute wählte. Als Beispiele mögen folgende Fälle dienen:

a) Schebester, Joh. liest am Beginne der Prüfung von Jaeger Nr. 16 nur das erste Wort "Einer"; nach 2 Minuten Präfungspause: "Einer neuen Wahrheit" ...; 1 Minute später: "Einer neuen Wahrheit ... nichts" ...; nach 1 Minute: "Einer neuen Wahrheit ... nichts ... als ein alter Irrthum". 
Nach 1 Minute (also 5 Minuten nach Beginn der Prüfung) vermag das Versuchsindividuam von Nr. 14 Alles zu lesen. Nach einer Pause von 5 Minuten erweist sich die Sehschärfe als unverändert.

b) Pechhaus. L. A. Nr. $16=\ldots$ W....; nach einer Minute $=\ldots$ W... schädlicher $\ldots$.. alter ...; nach 7 Minnten $=$ Einer $\ldots$ W... ist ... schädlicher ... alter Irrthum. Nr. $15=\mathrm{J} . \ldots$. Sch...; nach 5 Minuten: Nr. $16=$ Einer ... W.... ist .... schädlicher als.... Nr. $15=$ J. .. h.t ... Sch... Nach 2 Minuten, also 15 Minuten nach Beginn der Prüfung, Nr. $16=$ Einer ... W... heit ist ... schädlicher ... Irr...; nach 3 Minuten Nr. $16=$ E...W.... I... 5 Minuten später: Einer .... W...heit ist ... schädlicher als ... alter Irrthum; nach 7 Min. also $1 / 2$ Stunde nach Beginn der Prüfung, Nr. $16=\ldots$. W. . heit ist ... Irrthum. Im Verlaufe der folgenden 15 Minuten sinkt das Sehvermögen noch weiter herab, Nr. $16=\mathrm{E} . .$. . I....

c) Bij. R. A. Nr. $9=0$, von den einzelnen Buchstaben werden nicht einmal einzelne Striche wahrgenommen.

R. A. Nr. $10=\ldots$ Mensch $\ldots$ er ... da ... und ...

L. A. Nr. $10=\ldots$ kann der Mensch nicht .... daher schlägt er sich ... zu einer Parthei, weil er da ... nicht ... und Sicherheit findet. Nr. $9=\ldots$ sich im Ganzen ... und ... zurück. Nr. 8 $=$ Die ...

Die Sehprüfungen werden nunmehr nach Pausen von 3-5 Minuten und bei einer Prüfungsdauer von 1 Minute ausschliesslich an dem linken Auge angestellt.

4 Minuten später: L. A. Nr. $9=\ldots$ sich im Ganzen immer ... und ... nur durch ... zurück.

3 Minuten später: L. A. Nr. $9=\ldots$ sich im Ganzen immer vom Leben und ... nur durch einen Umweg ... dahin zurück.

3 Minuten später: L. A. Nr. $10=\ldots$ kann der Mensch nicht .... daher schlägt er sich ... zu einer Parthei, weil er da, wenn nicht Ruhe, doch ... and Sicherheit findet.

Nr. $9=\ldots$ sich im Ganzen immèr vom Leben und kehren nur durch einen Umweg wieder dahin zurück. $-\mathrm{Nr} .8=$ Die $\ldots$.

5 Minuten später ( $1 / 4$ Stunde nach Beginn der Prüfung): R. A. Nr. 10 $=\ldots$ der Mensch nicht $\ldots$ schlägt er ... er . . findet. - Nr. $9=0$.

L. A. Nr. $9=$ wie bei der vorausgegangenen Prüfung. $-\mathrm{Nr} .8=$ Die ... und ...

5 Minuten später: L. A. Nr. $8=$ Die ... und ... die ...

5 Minuten später: L. A. $\mathrm{Nr} .10=$ Alles. $\mathrm{Nr} .9$ wie bei der vorigen Prüfung. $N r .8=$ Die $\ldots$ als $\ldots$ and $\ldots$ aber $\ldots$ die $\ldots$

R. A. Nr. $10=\ldots$ der Mensch nicht ... schlägt er sich ... weil er da ... Nr. $9=\ldots$ and ... zurück ... 
Ueber $d$. Einfluss von Trigeminus-Reizen auf $d$. Sinnesempfindungen etc. 151

d) Schibek. 1. Versuch am 21./8.

Von Jaeger Nr. 10 wird nur die erste Zeile zur Prüfung benutzt; die Prüfung findet beim binoculären Sehen statt.

Nr. $10=$ All. . ... der M. . sch nicht ... schl.gt ...

5 Minuten später $=$ All...k... der Mensch nicht b.st.... d.h.. schl.gt $\ldots$.

10 Minuten später: = All. n kann der Mensch nicht best.h.. d.he. schlägt ...

Das Versuchsindividuum wird nunmehr angewiesen, das im Jaeger Nr. 10 vorkommende Wort "daher" genau zu fixiren; trotz des angestrengtesten Fixirens werden nur die Buchstaben d und he gelesen. Es wird hierauf eine Lufteinblasung in beide Ohren vorgenommen. Unmittelbar danach treten die Buchstaben e und $r$ deutlich hervor, so dass nun das Wort "daher" vollständig lesbar erscheint. Binnen 5 Sekunden schwindet jedoch die Besserung des Sehvermögens und es bleiben wiederum nur die Buchstaben d und he sichtbar. Dasselbe ergibt eine unmittelbar darauf ausgeführte Lufteinblasung. Es werden nunmehr die Sehprüfungen weiter fortgesetzt; nach 5 Minuten wird das Wort "daher" ohne etwa ausgeübte Luftdouche gelesen und bleibt von diesem Momente an, wie die späteren Sehprüfungen ergeben, gleichmässig deutlich sichtbar.

2. Versuch. 25./8.

L. A. Nr. $11=$ Von N.... Nr. $10=0$.

Das rechte Auge vermag Nr. 8 theilweise zu lesen, von Nr. 7 nur einzelne Worte, von Nr. 6 (dessen erster Satz folgendermassen lautet: „Mensch, herrliche, hohe Erscheinung!") gar nichts. Bei fortgesetzten Sehprüfungen (in Intervallen von 2-4 Minuten) tritt 18 Minuten nach Beginn des Versuches von Nr. 6 rom ersten Worte "Mensch" der Buchstabe M als B hervor, nach 2 Minuten B..ch, nach 3 Minuten B..ch ...ich. 30 Minuten nach Beginn der Prüfung liest das bisher in den Versuch nicht einbezogene linke Auge von $\mathrm{Nr} .11=$ Vo. N... ... wir ... der ... zur ...; von Nr. $10=\mathrm{A} \ldots$.

Die mit dem rechten Auge wieder allein fortgesetzten Prüfungen ergaben: 5 Minuten nach dem letzten. Versuche, Nr. $6=\ldots$ e.ch ... sch.. g... Nach 2 Minuten später $=$ Mensch, ... sch. allen ... (,allen" aus dem 2. Satze von Nr. 6). 1 Stunde nach Beginn der Prüfung mit dem rechten Auge vermag das linke A uge zu lesen: Nr. $10=$ All.. kann der Mensch nicht besteh... daher schlägt .....

Wie den mitgetheilten Beispielen zu entnehmen ist, bewirkt, ein angestrengtes Sehen in manchen Fällen eine Steigerung des Sehvermögens, ein andermal dagegen eine vorübergehende $\mathrm{Ab}$ nahme oder aber Schwankungen der Sehstärke. Betreffs der stärkeren Erregung des Gesichtssinnes wäre zu bemerken, dass bierzu 
keineswegs continuirliche Sehimpulse nöthig sind, sondern dem Auge zeitweise zugeführte Reize sich als rollständig genügend erweisen.

Ja selbst bei Einschaltung von Prüfungspausen in einer Daner von 5-10 Minuten gibt sich, den angefübrten Beobachtungen zafolge, eine stetige Steigerung des Sehvermögens manchmal deutlich zu erkennen. Dieselbe erscheint dabei zuweilen als ziemlich erheblich und betrug in einzelnen der mitgetheilten Fälle $1 \mathrm{Nr}$. der Jaeger'schen Schriftskalen und sogar noch darüber. Die Schnelligkeit, mit der das Sehvermögen ansteigt, ist individuell sehr versehieden, so dass eine bestimmte Sehbesserung in einem Falie binnen 10 Minuten, in einem anderen Falle vielleicht erst nach einer Stunde erreicht wird.

Wie aus den mitgetheilten Versuehsergebnissen klar hervorgeht, ist die Sebbesserung eine wirkliche and nicht scheinbare. Wenigstens lässt sich das während der Sehprüfungen allmähliche Hervortreten von früher nicht wahrnehmbaren Buchstaben keineswegs so erklären, als ob das untersuchte Auge bei wiederholten Prüfungen von den bereits früher gelesenen Buchstaben absieht und seine volle Aufmerksamkeit den bisher nicht wahrnehmbaren Buchstaben zuwendet und diese dadurch allmählich zu erkennen vermag. Dagegen spricht nämlich die Thatsache, dass am Beginn der Prüfung, von einer bestimmten Schriftskala, trotz des angestrengtesten Sehens, nicht ein einziger Buchstabe wahrnehmbar sein kann, während im Verlaufe der Untersuchung, bei vollständig gleich bleibender Versuchsanordnung, von derselben Nr. Worte ja sogar Sätze gelesen werden können, wenn das Auge mittlerweile wiederholt zum angestrengten Sehen genöthigt wurde. Im Falle c) fand die Priufung vorzugsweise mit Jaeger Nr. 9 und Nr. 8 statt, von welchen einzelne Silben gelesen wurden, indess Nr. 10, von der die Versuchsperson anfänglich einzelne Worte wahrzunehmen vermochte, nur zeitweise in Verwendung kam; trotzdem erschien die Sehsteigerung eben für Nr. 10 am auffälligsten. Für die Annahme, dass zeitweise zugeführte Sehimpulse thatsächlich eine Steigerung des Gesichtssinnes bedingen, spricht vor Allem die Beobachtung einer Sehsteigerung am nicht geprüften Auge, welche durch die Erregung der Gesichtsempfindung: am anderen Auge hervorgerufen wird.

Am auffälligsten tritt diese Erscheinung im Falle d) entgegen, 
in welchem die durch eine Stunde ausschliesslich am rechten Auge angestellten Sehproben, am linken Auge eine relativ erhebliche Steigerung des Sehvermögens erzielten. Ich muss hier besonders betonen, dass die Sehsteigerung am nicht geprüften Auge auch dann stattfindet, wenn dieses der gleichen Lichteinwirknng ausgesetzt ist, wie das geprüfte Auge, demzufolge die am nicht geprüften Auge erfolgende Sehzunahme nieht etwa einer in der Dunkelheit stattfindenden allmäblichen Erholung des betreffenden Sehorganes zugeschrieben werden kaun.

Beim monoculären Sehen findet also eine Erregung der optischen Zentren statt, welche eine Steigerung des Sehvermögens an beiden Augen zur Folge hat.

Als eine dieser ganz analogen Erscheinung ist die Beobachtung von Volkmann ${ }^{1}$ ) anzuführen, der zufolge eine durch Uebung herbeigeführte Verfeinerung des Ortsinnes der Haut an einer bestimmten Körperstelle, auch an der correspondirenden Stelle der anderen Körperseite einen verfeinerten Ortsinn ergibt. Auch die von C. H. Weber ${ }^{2}$ ) beobachtete Mitübung symmetrischer Maskeln ist hierher zu beziehen.

Betreffs ähnlicher Erscheinungen des Gehörsinnes behalte ich mir weitere Mittheilungen vor.

Der Nachweis einer spontan erfolgenden Sehsteigerung mahnt zur Vorsicht, dass man die nach einer Erregung des äusseren oder mittleren Ohres eintretende Besserung des Sehvermögens nicht dieser Erregung allein zuschreibe. Es drängt sich daher die Frage auf, ob man uiberhanpt die direkt zu Stande gekommene Sehbesserung von einer indirekt ausgelösten unterscheiden könne. Bei Durehsicht der verschiedenen Versuchsergebnisse findet sich, wenigstens im Allgemeinen, ein solcher Unterschied wohl vor, und zwar gibt sich die rasch eintretende und gewöhnlich binnen wenigen Minuten vorübergehende Besserung der Sehfunktion als eine Eigenthümlichkeit der indirekt ausgelösten Erregung zu erkennen, wogegen die allmählich ansteigende und je nach der Dauer der Prüfung, durch 1/2-1 Stunde und dariuber, stetig zunehmende Intensität der Sehempfindung eher als ein Merkmal der spontan zu Stande gekommenen Sehbesserung anzusehen ist.

1) Ber. d. sächs. Gesellsch. d. Wissensch. 1858.

2) s. Funke in Hermann's Handbuch der Physiol. 1880, Bd. III, Th. 2, p. 382. 
Es wäre diesbezüglich auf den Fall d) besonders aufmerksam zu machen. Das betreffende Versuchsindividuum konnte nämlich in einer gewissen Phase des spontan zunehmenden Sehvermögens von dem in Nr. 10 der Jaeger'schen Schriftskala befindlichen Worte „daher", nur die Buchstaben d und he lesen. Nach einer Lufteinblasung ins Mittelohr erschienen anch die vorher nicht wahrnehmbaren Buchstaben a und $r$, jedoch nur auf 5 Sekunden, so dass also während dieser Zeit das Wort "daher" deutlich gesehen wurde, dagegen 5 Sekunden später nur d und he ${ }^{1}$ ).

Ein unmittelbar danach angestellter 2. Versuch hatte dasselbe Resultat aufgewiesen. Während der weiter vorgenommenen Priifung war die spontan zunehmende Besserung des Sehvermögens, 5 Minuten nach dem letzterwähnten Versuche weiter vorgeschritten, so dass nunmehr das Wort „daher" vollständig hervortrat, ohne jedoch diesmal wieder zurückzugehen. Das direkt ansteigende Sehvermögen hatte also nach 5 Minuten die Höbe erreicht und auch beibehalten, welche die indirekt erhöhte Sehfunktion, 5 Minuten vorher, nur auf wenige Sekunden einzunehmen im Stande war.

Da durch die angegebenen Untersuchungen die Möglichkeit und sogar Häufigkeit einer Beeinflussung des Sehvermögens durch die verschiedenen Eingriffe auf das äussere und mittlere $\mathrm{Ohr}$ sichergestellt erschien, musste nunmehr entschieden werden, worauf die nachgewiesene Veränderung des Sehvermögens beruhe, ob sie mit gewissen sichtbaren Veränderungen im Augenhintergrunde einhergehe oder nicht.

In dieser Beziehung wäre vor Allem hervorzuheben, dass, wie einige Fälle Zaufal's lehren, bei Entzündungen des Mittelobres eine Ausbreitung der entzündlichen Erseheinungen auf die Retina möglich ist. Eine Yeränderung der Retina liess sich jedoch bei den von mir beobachteten Individuen mit herabgesetzter Sehstärke, nur für jene Fälle muthmassen, in denen mehr minder beftige Entzündungen des änsseren oder mittleren Ohres bestanden.

1) Zu solchen Versuchen eignen sich selbstverständlich nur intelligente und besonders eingeübte Personen, welche die genügende Objectivität besitzen, von einem ihnen etwa bekannten Worte nur jene Buchstaben anzugeben, die sie thatsächlich sehen:

2) Prager med. Wochenschr. 1881, Nr. 45; s.ferner Politzer, Ohrenheilk., p. 654. 
Dagegen erschien es sehr unwahrscheinlich, dass Affektionen des Ohres, welche ohne die geringste nachweisbare Hyperaemie einhergehen, Veränderungen der Retinalgefässe herbeizuführen vermögen; auch die Beobachtung von plötzlich eintretender bedeutender Sehbesserung, sowie die bei einseitiger Onrenbehandlung am Auge der anderen Seite ausgelöste Sehsteigerung, sprachen gegen die Annahme einer vom Ohre aus auf die Retina ïbergetretenen Hyperaemie. Betreffs des letzteren Umstandes muss allerdings bemerkt werden, dass in den Fällen Zaufal's, bei einseitiger Mittelohrentziundung, auch an der Retina des gegenseitigen Auges Reizerscheinungen vorgefunden wurden.

Zur Lösung dieser Frage hatte Herr Dr. Bergmeister, Dozent für Augenheilkunde an der Universität in Wien, die Güte, an verschiedenen Patienten mit einfachem Mittelohrcatarrh, eitriger Entzündung der Paukenhöhle und Obrpolypen, ophthalmoskopische Untersuchungen anzustellen und zwar wurden die Patienten mit dem Augenspiegel vor und nach der Ohrenbehandlung untersucht. Es ergab sich hierbei, dass selbst an solchen Individuen, bei denen die Ohrenbehandlung, oder ein einzelner Eingriff auf das äussere bez. mittlere Ohr, eine wesentliche Steigerung des Sehvermögens erzielt hatte, der Augenspiegelbefund vollständig unverändert geblieben war. Erwähnenswerth ist ferner noch der Befund, dass in den von Herrn Dr. Bergmeister untersuchten Fällen von Ohrpolypen und eitriger Entzünảung der Paukenhöhle, keine $\mathrm{Hy}$ peraemie der Retinalgefässe nachgewiesen werden konnte. Wenngleich sich die ophthalmoskopischen Untersuchungen nicht auf sämmtliche hier angeführte Ohrenkranke, sondern uur auf einen kleinen Theil derselben bezogen, so muss doch besonders betont werden, dass eben in solchen Fällen der Augenspiegelbefund aufgenommen wurde, in denen die Veränderungen des Sehvermögens sehr beträchtliche Schwankungen aufwiesen. Jedenfalls war damit der Nachweis erbracht, dass vom Ohre ans Veränderungen des Sehvermögens ausgelöst werden können, die ohne die geringsten ophthalmaskopisch sichtbaren Veränderungen im Augenhintergrunde einhergehen.

Es musste nun direkt zur Ermittlung der Frage geschritten werden, wodureh die Aenderung der Sehfunktion bei den erwähnten Versuchspersonen zu Stande komme. Das Erkennen von Sehobjekten hängt bekanntlich nicht bloss von der Schärfe und Grösse 
des Netzhautbildes, sondern auch von der Empfindlichkeit des Opticus-Apparates ab. Bei unseren Versuchsindividuen war schon im Vorhinein anzunehmen, dass die das 0 br treffenden Reize nur diese letztere zu beeinflussen vermögen.

Ich hatte daher durch weitere Untersuchingen festzustellen, ob sich bei den versebiedenen Einwirkungen anf das Ohr, am Lichtsinne irgend welche Intensitätsschwankungen nachweisen lassen.

Die Angabe vieler Versuchsindividuen, dass bei irgend einer Reizeinwirkung auf das Obr das Gesichtsfeld heller und später wieder dunkler werde, sprach schon im Vorhinein für die Wahrscheinlichkeit derartiger Schwankungen. Als Beispiele mögen folgende Fälle dienen: Ein Versuchsindividum (Alb. Stieglitz) erkannte von der Jaeger'schen Schriftskala Nr. 12 keinen Buchstaben, sondern sah dieselbe nur als graue Hläche. Im Momente der Einfiibrung eines Tampons ins linke Obr hellte sich das Gesichtsfeld auf, die Striche der Buchstaben traten deutlich hervor, ohne dass jedoch ein Buchstaben gelesen werden konnte. Nach 45 Sekunden begann die Verdunklung des Gesichtsfeldes und erreichte binnen weiteren 45 Sekunden den ursprünglichen Standpunkt. Nach einer Ausspritzung des Ohres erfolgte eine abermalige Aufhellung; diese begann 8 Sekunden nach der Ausspritzung; nabm durch 2 Sekunden weiter zu, ging 35 Sekunden später wieder zuriick and erschien nach 10 Sekunden vollständig geschwunden. Eine Lufteinblasung in beide Paukenhöhlen, mittelst des Politzer'schen Verfahrens, hellte nach 8 Sekunden das Gesichtsfeld abermals auf, 35 Sekunden später wurde (von Jaeger Nr. 12) der Buchstabe $S$ gesehen, derselbe sehwand jedoch nach einigen Sekunden, bei eintretender Verdunkelung des Gesichtsfeldes.

Behufs genauerer Prüfungen des Lichtsinnes, welche ich an einer Reihe von Individuen in den verschiedenen Stadien der Ohrenerkrankung, ferner nnmittelbar vor und nach einer Einwirkung auf das äussere oder mittlere Ohr anstellte, bediente ich mich eines Photometers nach Förster, welches mir Herr Professor v. ArIt gutigst iberlassen hatte. Die einzelnen Untersuchungen fanden jedesmal nach einem, wenigstens 5 Minuten währenden Aufenthalt der betreffenden Versuchsperson in der Dunkelkammer statt. Am Beginne der Untersuchung wurde die anfänglich auf 0 eingestellte Photometer-Schraube sehr langsam gedreht, bis das 
geprifte Auge einzelne schwarze Streifen von der an der hinteren Wand des Photometer-Kastens angebrachten Tafel (die 8 Streifen, je 4 über einander gestellt enthält) bemerkte. Sobald die Versuchsperson angab, einzelne Streifen zu erblicken, wurde mit der weiteren Drehung der Schraube innegehalten und durch wiederholte Prüfungsversuche (bei Einschaltung von Ruhepansen) die Anzahl der sichtbaren Streifen genau bestimmt. Bei manchen Personen erwiesen sich solehe Controllversuche sehr nöthig, indem bei gleicher Lichtstärke von der Photometer-Tafel, besonders im Anfange des Versuches, bald nur ein Strich, bald mehrere Striche abgelesen wurden. Verschiedene Versuchspersonen mussten sogar von den weiteren Prüfungen vollständig ausgeschlossen werden, da bei ihnen beträchtliche Schwankungen des Lichtsinns unaufhörlich stattfanden. So geschah es zu wiederholten Malen, dass eine solche Versuchsperson, trotz der zwischen die einzelnen Versuche eingeschalteten gleich grossen Pausen und gleicher Dauer der Einzelprüfung, bald nur einzelne, bald sämmtliche 8 Striche an der Photometertafel erblickte.

Die Sehproben stellte ich stets an jedem Auge abgesondert an und achtete dabei sorgfältigst, dass auf das von der Prüfung ausgeschlossene Auge nicht der geringste Druck (seitens der das Auge verschliessenden Hand etc.) ausgeübt wurde. Wie ich nämlich an mir zuerst beobachtete, vermag ein oft geringfügiger Druck auf das eine Ange den Lichtsinn am anderen Auge mächtig zu beeinflussen. Wenn ich beispielsweise mit meinem rechten Auge einzelne Striche an der Photometertafel deutlich und gleichmässig gut sehe und hierauf auf den linken Bulbus einen nur schwachen Druck ausiibe, so erscheint unmittelbar darauf oder binnen wenigen Sekunden der Lichtsinn am rechten Auge bedeutend herabgesetzt, die Streifen werden undentlich und schwinden binnen Kurzem vollständig; bei fortwährend anhaltendem Drucke auf das linke Auge tanchen dem rechten Auge ein oder mehrere Striche an der Photometertafel hervor und schwinden in der nächsten Sekunde, um später wieder zu erscheinen etc. Sobald ich mit dem Druck am linken Auge nachlasse, geht das beschriebene Phänomen am rechten Auge rasch zurück und der Lichtsinn weist seine fribere Intensität auf. Eine derartige Beeinflussung des Lichtsinnes an einem Auge durch Druck auf das andere Auge konnte ich bei vielen Versuchspersonen bald mehr, bald minder deutlich nach- 
weisen. Während dieser also herbeigeführten Herabsetzung des Lichtsinnes lassen sich an meinem Auge, wie dies mehrere an mir angestellte Untersuchungen des Herrn Dr. Bergmeister's ergaben, keine Veränderungen am Augenhintergrunde erkennen.

Die unter den erwähnten Cautelen an verschiedenen Versuchspersonen angestellten photometrischen Untersuchungen ergaben folgendes:

A. Pren; links Polyp der Paukenhöhle.

a) R. A. Nr. 16 ausser "Wahrheit" alles.

L. A. Nx. $17=$ Liebe versch....

Photometer bei $2 \mathrm{~mm}$ Schraubeneinstellung:

R. A. = oben 4 Striche, upten 4 Striche.

L. A. $=3$ Striche in gleicher Linie.

ß) 7 Tage später nach Extraction des Polypen.

R. A. Nr. $16=$ Alles; Nr. $15=J$. höh.. ... Menschen .. zu..

L. A. Nr. $17=$ Alles; Nr. $16=\ldots$ ist nicht $\ldots$ ein $\ldots$

Photometer bei $2 \mathrm{~mm}$ Schraubeneinstellung:

R. A. = oben 4 Striche, unten 4 Striche.

L. A. $=$ oben 4 Striche, unten 4 Striche.

Bei $1 \mathrm{~mm}$ :

R. A. $=$ oben 3 Striche, unten 2 Striche.

L. A. $=$ oben 3 Striche, unten 2 Striche. Otarrhoe:

y) 1 Woche später nach bedeutender Besserung der früher profusen

R. A. Nr. $16=$ Alles; Nr. $15=$ J. h. . ... steht ... Menschen ..

L. A. Nr. $16=$ Einer $\mathrm{n} \ldots$ ist nichts schädlicher als ein alter Irrthum. - Nr. $15=$ J. ...

Photometer, $1 \mathrm{~mm}$ :

R. A. = oben 3 Striche, unten 2 Striche.

L. A. = oben 3 Striche, unten 3 Striche.

B. Schafer, Rud.; rechts 6 Mon. Polyp der Paukenböhle. Bilateral hochgradige Hypermetropie $1 / r$, links Centralkapselstarr (Dr. Bergmeister).

a) R. A. Nr. $24=0 .-$ L. A. Nr. $18=$ Alles; Nr. $17=0$.

Photometer, $2 \mathrm{~mm}$ :

R. A. $=1$ Strich. - L. A. $=2$ Striche.

в) 2 Tage später: R. A. Nr. $24=0 .-$ L. A. Nr. $17=$ Liebe $\ldots$. Photometer, $1 \mathrm{~mm}$ :

R. A. $=2$ Striche - L. A. $=4$ Striche oben, 4 Striche unten.

Unmittelbar nach einer Lufteinblasung in beide Ohren:

R. A. Nr. 24, 23 und 22, nach 3 Minuten auch Nr. $21=$ Alles.

L. A. Nr. $17=$ Liebe $\ldots .$. . 
Ueber d. Einfluss von Trigeminus-Reizen auf d. Sinnesempfindungen etc. 159

Photometer, $1 \mathrm{~mm}$ :

R. A. $=3$ Strich. - L. A. $=4$ Striche oben, 4 Striche unten.

y) Einige Tage später:

R. A. Nr. $21=$ Danzig. - L. A. Nr. $17=$ Liebe verschenkt $\ldots$ Photometer, $5 / 4 \mathrm{~mm}$ :

R. A. = bald 1 Strich, bald 2 Striche.

L. A. = bald 3 Striche, bald 4 Striche.

C. Schibek. Catarrh des rechten Mittelohres. Rechts Hypermetropia tot. geringgradig ca. ${ }^{1 / 40}$, oben Conus; links Hypermetr. tot. ca. $1 / 16$; ein kleines Staphylom nach unten (Dr. Bergmeister).

R. A. Nr. $14=$ Der allein ist glücklich und gross, der weder zu...

L. A. Nr. $16=$ Einer neuen Wahrheit ....

Photometer, $1 \mathrm{~mm}$ :

R. A. = oben 2 Striche, unten 1 Strich.

L. A. $=1$ Strich.

Nach Catheterisation des rechten Tubenkanales:

R. A. Nr. $14 \rightleftharpoons$ Der allein ist glücklich und gross, der weder zu .... und zu ge......

L. A. Nr. $16=$ Einer neuen Wahrheit ist ... als ein alter Irrthum. Photometer, $1 \mathrm{~mm}$ :

R. A. = oben 2 Striche, unten 1 Strich.

L. A. = oben 1 Strich, unten 1 Strich.

D. Beyer. Seit 2 Monaten rechts eitrige Entzündung der Paukenhöhle. Photometer, $3 / 4 \mathrm{~mm}$ :

R. A. = oben 1 Strich, unten 1 Strich.

L. A. $=$ oben 4 Striche, unten 4 Striche.

Nach einer Lufteinblasung in beide Obren:

K. $A$. = oben 3 Striche, unten 1 Strich.

L. $A$. = oben 4 Striche, unten 4 Striche.

Nach 3 Minuten geht die Besserung am rechten Auge vorüber.

E. Böhm. Bilateraler chron. Mittelohrcatarrh; Uhr rechts $6 \mathrm{~cm}$, links $1 \mathrm{~cm}$ weit gehört.

*) Photometer, $3 \mathrm{~mm}$ :

R. A. $=4$ Striche. - L. A. $=3$ Striche.

Bei $2 \mathrm{~mm}$ Schraubeneinstellung:

R. A. $=1$ Strich. - L. A. $=0$.

в) Einige Tage später:

Photometer bei $2 \mathrm{~mm}$ und bei $3 \mathrm{~mm}$ :

R. A. =0. - L. A. $=0$.

$4 \mathrm{~mm}$ :

R. A. $=0 .-$ L. A. $=2$ Striche; unmittelbar darauf:

R. A. $=3$ Striche. - L. A. $=3$ Striche. 
$3 \mathrm{~mm}$ :

R. A. = 1 Strich. - L. A. = 2 Striche.

$2 \mathrm{~mm}$ :

R. A. $=2$ Striche. - L. A. $=2$ Striche.

F. Janusch. Links eitrige Entzündung der Paukenhöhle. Von Jaeger Nr. 16 vermag das rechte Auge mehr Buchstaben zu lesen als das linke. Photometer, $1 \mathrm{~mm}$ :

R. A. = oben 4 Striche, unten 4 Striche.

L. A. = oben 4. Striche, unten 3 Striche.

Unmittelbar danach:

R. A. = oben 4 Striche, unten 3 Striche.

L. A. = oben 3 Striche, unten 2 Striche.

Nach Vornahme des Politzer'schen Verfahrens:

R. A. $=$ oben 4 Striche, unten 2 Striche.

L. A. = oben 4 Striche, unten 3 Striche.

G. Pschiwanek. Rechts eitrige Entzündung der Paukenhöhle.

a) Photometer, $5 / 4 \mathrm{~mm}$ :

R. A. = oben 4 Striche, unten 2 Striche (undeutlich).

L. A. = oben 4 Striche, unten 2 Striche (deutlich).

Nach Politzer'schem Verfahren:

R, A. $=$ ohen 4 Striche, unten 3 Striche (undeutlich).

L. A. = oben 4 Striche, unten 3 Striche (deutlich).

Nach 2 Minuten sinkt der Lichtsinn, rechts schneller als links.

B) 2 Tage später. Photometer, $5 / 4 \mathrm{~mm}$ :

R. A. $=0 .-$ L. A. $=0$.

Nach Politzer'schem Verfahren:

R. A. = oben 3 Striche, unten 2 Striche.

L. $A$. $=$ oben 3 Striche, unten 2 Striche.

H. Bij. Links eitrige Mittelohrentzündung.

R. A. liest Nr. 17 theilweise; L. A. Nr. 16 theilweise.

Photometer, $1 \mathrm{~mm}$ :

R. A. = oben 4 Striche, unten 4 Striche.

L. A. $=0$.

Durch die Guite des Herrn Professors Stellwag wurde mir die Gelegenheit geboten, an mehreren Patienten mit abnorm herabgesetztem Lichtsinn, den Einfluss von Lufteintreibungen ins Mittelohr und von Bougirung des Tubenkanales auf den Lichtsinn zu prüfen.

Die Fälle sind nachfolgende: 
Ueber d. Einfluss von Trigeminus-Reizen auf d. Sinnesempfindungen etc. 161

1) Ein Fall von Neuroretinitis.

Bei $28 \mathrm{~mm}$ Schraubeneinstellung wird 1 Streifen sehr undeutlich gesehen. Eine Bougirung des linken Tubenkanales und Lufteinblasungen ins Ohr bewirken eine bedeutende Aufbellang, 1 Minute später treten 2 Streifen, 15 Minuten darauf noch ein 3. Streifen deutlich hervor ${ }^{1}$ ). Die Schraube wird auf 0 eingestellt und langsam gedreht; es erscheinen nunmehr bereits bei $25 \mathrm{~mm} 3$ Streifen. 5 Minuten nach der Lnfteinblasung sinkt allmäblig der Lichtsinn.

2) Fall von Glaucoma simplex dextrum.

Es wird nur das erkrankte rechte Auge photometrisch geprüft.

Bei $5 \mathrm{~mm}$ Einstellung bemerkt die Patientin $1 \frac{1}{2}$ Streifen sehr undeutlich, nach der Catheterisation des rechten Tubenkanales dagegen 3 Streifen; so auch bei einem 2. and 3. Versuch.

3) Fall von Retino-Chorioiditis; Pigmentatrophie der Netzhaut.

Bei $45 \mathrm{~mm} 1$ Streifen; Lufteintreibungen ins Ohr bewirken keine Aufhellung des Gesichtsfeldes.

1) Bezüglich des Auftretens mehrerer Streifen bei gleichbleibender Finstellung der Photometer-Schraube möchte ich hervorheben, dass wegen der in den Photometer-Kasten seitlich einfallenden Lichtstrablen, am Photometer nach Förster, die Beleuchtung der hinteren Wand keine gleichmässige ist, sondern die der Lichtquelle direkt gegenüberstehende Theile der Tafel intensiver beleuchtet werden als die anderen Abschnitte derselben, demzufolge die Streifen an der bezeichneten Stelle, sowie in deren Nähe zuerst sichtbar sind; ferner wäre noch zu bemerken, dass die Streifen von verschiedener Dicke sind und die dickeren Streifen meistens vor den dünneren hervortreten.

Ich habe weiters zu erwähnen, dass ich bei den bisher angeführten photometrisch Untersuchten mit der Schraubendrehung stets in dem Momente innehielt, in welchem die Versuchsperson einen oder einzelne Streifen erblickte, indess in den zuletzt erwähnten Fällen (s. unten) die Schraube solange gedreht wurde, bis der für die einfallenden Lichtstrahlen bestimmte quadratische Ausschnitt an der vorderen Wand des Photometer-Kastens eine Grösse erreichte, welche jene Lichtmenge in den Photometer-Raum eindringen liess, bei der dem betreffenden Versuchsindividuum sämmtliche 8 Streifen sichtbar erschienen. Behufs vergleichsweiser Prüfungen stellte ich stets die Photometer-Schraube rasch auf 0 ein und drehte sie allmählich bis zu jener Einstellung, bei der sämmtliche 8 Striche hervortraten. Den einzelnen Fällen sind die Grösse der Lichtpforte in Quadratmillimetern und die daraus berechnete Stärke des Lichtsinnes (die Einstellung von $2 \mathrm{~mm}$ als 1 angenommen) beigegeben (vergl. Ma uthner, Augenheilk. 1879, Heft 3, S. 147). 
4) Fall von Atrophia nerv. optic. dextr.; keine Ursache nachweisbar.

Bei einer Schraubeneinstellung von $30 \mathrm{~mm}$ (Grösse der Lichtpforte $450 \square \mathrm{mm}$ ) werden alle 8 Striche gesehen (Lichtsinn 1/225); unmittelbar nach Lufteinblasungen ins rechte Mittelohr erblickt das rechte Auge bei $20 \mathrm{~mm}$ $(200 \square \mathrm{mm})$ die 8 Streifen $(\mathrm{L}=1 / 100), 8$ Minuten später bei $18 \mathrm{~mm}(162 \square \mathrm{mm})$ dieselben Streifen $(\mathrm{L}=1 / 81$, nach 2 Minuten bei $15 \mathrm{~mm}(1121 / 2 \square \mathrm{mm}$, Licht$\operatorname{sinn}=1 / 56$ ); 10 Minuten nach der Einblasung erscheinen bereits bei $10 \mathrm{~mm}$ $(50 \square \mathrm{mm})$ die 8 Streifen $(\mathrm{L}=1 / 25) .15$ Minuten nach der Einblasung beginnt der Lichtsinn allmählich zu sinken.

5) Fall von Entfärbung der Sehnerven mit muldenförmiger Excavation (rechts eine kleine Haemorrhagie in der Papille), Verdacht auf Glaucom; ausgesprochene hemeralopische Erscheinungen.

Bei der Maximaleinstellung von $50 \mathrm{~mm}(1250 \square \mathrm{mm})$ nimmt die Patientin keinen einzigen Strich aus. Unmitteibar nach der Catheteterisation des linken Ohres erscheinen 2 Streifen, nach 5 Minuten 4 , nach 8 Minuten 5 Streifen; 10 Minuten nach dem Catheterismus erblickt die Patientin sämmtliche 8 Streifen (Lichtsin $={ }_{1625}^{1}$ ); 5 Minuten später werden bereits bei $33 \mathrm{~mm}$ $\left\langle\left. 544^{1}\right|_{2} \square \mathrm{mm}\right)$ alle 8 Streifen gesehen $\left(\mathrm{L}=\left.\right|_{272}\right)$. Hierauf beginnt der Lichtsinn rasch zu sinken.

6) Fall von Decoleratio nerv. optic. ocul. utr.

Bei $3 \mathrm{~mm}\left(4_{1}{ }_{2} \square \mathrm{mm}\right)$ werden alle 8 Streifen $\left(\mathrm{L}=\left.{ }^{1}\right|_{2}\right)$ nach Lufteinblasungen ins $O \mathrm{hr}$, bei $2 \mathrm{~mm}(2 \square \mathrm{mm})$ sämmtliche Streifen $(\mathrm{L}=1)$ gesehen. 10 Ninuten später nimmt Patient die 8 Streifen, wie beim Beginne der Untersuchung, erst bei einer Schraubeneinstellung von $3 \mathrm{~mm}$ wahr.

In den angeführten Beispielen fand sich ein stärker entwickelter Lichtsinn in der Regel an dem Auge vor, welches die Schriftproben besser zu lesen vermochte, nur der Fall $H$ macht eine bemerkenswerthe Ausnahme davon. Wie aus den oben erwähnten Versuchen hervorgeht, erweist sich die Zunahme des Sehvermögens für die Schriftskalen sehr häufig unverhältnissmässig bedeutender als dies bezüglich des Lichtsinnes der Fall ist (s. Fall B); es ergibt sich ferner, dass einem bestimmten Sehvermögen für die Schriftskalen nicht immer ein bestimmter Grad des Lichtsinnes entspricht (s. Fall A $\beta$ ), sowie anch bei einem bedeutenden Unterschied des Sehrermögens am rechten und linken Auge, ein annähernd gleich stark entwickelter Lichtsinn oder wenigstens ein bei weitem geringerer Unterschied der Intensität des Lichtsinnes an beiden Augen vorkommen kann (s. Fall $\mathrm{B} \alpha$ ). Bei den erheblichen Schwankungen des Lichtsinnes wird man allerdings einer einzelnen der. 
Ueber d. Einfluss von Trigeminus-Reizen auf d. Sinnesempfindungen etc. 163

artigen Erscheinung keine besondere Bedeutung beimessen können. Viel verlässlicher sind die Untersuchungs-Ergebnisse hinsichtlich der Beeinflussung des Lichtsinnes durch verschiedene auf das äussere oder mittlere Ohr ausgeübte Reize; so ist die Erhöhung des Lichtsinnes nach einer Lufteinblasung ins Mittelohr meistens eine beträchtliche und zeigt sich, wie mich viele Versuche lehrten, keineswegs nur bei Ohrenkranken, sondern kommt auch bei vollständig gesunden Individuen vor. Der Effekt ist allerdings zuweilen nur ein momentaner und tritt dann gewöhnlich unmittelbar nach der Reizeinwirkung auf das Ohr hervor.

Betreffs der ïbrigen an den photometrisch Untersuchten beobachteten Erscheinungen wäre nur in kurzem auf die Uebereinstimmung derselben mit den bei den Sehprüfungen mittelst Schriftskalen angestellten Beobachtungen hinzuweisen. So erfolgt zuweilen nach einer Irritation des äusseren oder mittleren Ohres anstatt einer Erhöhung eine Herabsetzung des Lichtsinnes u. z. sogar bei demselben Individuum; es sind ferner anzuführen, eine, bei einseitiger Einwirkung auf das Ohr, an beiden Augen eintretende Veränderung des Lichtsinnes, die ungleich starke oder verschieden lange anhaltende Steigerung desselben am rechten und linken Auge, die durch einige Zeit wellenartig auf- und niederwogende Intensität des Lichtsinnes, der bei wiederholt zugeführten Reizen immer schwächer werdende Effekt, endich die Steigerung des Lichtsinnes nach seiner einmal erfolgten Anregung. Diese letztere Erscheinung fand ich besonders schön an einem Kollegen vor, der im Beginne der photometrischen Untersuchung bei einer Schraubenstellung von 2, 3, 4 bis $5 \mathrm{~mm}$, trotz des angestrengten Fixirens, an der Photometertafel nicht einen einzigen Strich, weder mit dem rechten noch mit dem linken Auge, walırzunehmen vermochte, dagegen bei einer weiteren Drehung der Schraube plötzlich sämmtliche 8 Striche sah, dann aber diese, trotz des raschen Zurückdrehens der Schraube bis auf $1 \frac{1}{2} \mathrm{~mm}, \mathrm{zu}$ unterscheiden im Stande war und auch später, noch bei der bezeichneten Schraubeneinstellung, sämmtliche Striche erkannte (vgl. ferner Fall 1, S. 159$\rangle^{1}$ ).

Durch alle die oben besprochenen Untersuchungs-Ergebnisse erschien es also sichergestellt, dass vom äusseren und mittleren Ohre aus eine Beeinflussung des Sehvermögens vermittelst einer

1) Vgl. S. 131. 
Einwirkung auf den Lichtsinn erfolgt, und dass ferner, da Lichtsinn and Sehvermögen nicht immer einander parallel gehen, noch ein weiterer, vorläufig ganz räthselhafter Umstand den angefuhrten Erscheinungen zu Grunde liegen muss. In Bezug auf den ersten Punkt blieb nunmehr die Frage zu lösen, worauf eine derartige Einflussnahme des Gehörorgans auf den Lichtsinn beruhe.

Wenngleich eine Hebung der Sehstärke gewöhnlich im Gefolge einer Steigerung der Hörfunktion angetrofien wurde, so liessen doch die Untersuchungen leicht erkennen, dass das Sehvermögen von der Hörfähigkeit vollständig unabhängig sei. Wie nämlich die Erfahrungen an Ohrenkranken lebren, kann irgend ein therapeutischer Eingriff auf das $\mathrm{Ohr}$ allerdings gleichzeitig eine Besserung des Gebörs und des Sehvermögens bewirken, die Sehbesserung geht jedoch gewöhnlich sehr rasch zurück, indess die gesteigerte Hörfähigkeit unverändert anbält, oder aber das anfänglich gebesserte Gehör sinkt wieder zu seiner früheren Stufe herab, ohne dadurch das Sehvermögen irgend wie zu beeinträchtigen. So traf ich ferner mehrere Ohrenkranke an, bei denen der Eingriff auf das Ohr, gleich im Anfange der Behandlung, eine plötzlich auftretende beträchtliche Sehverbesserung erzielte, wogegen zu derselben Zeit der Zustand des Gehörs nur eine unbedeutende Veränderung aufwies und auch im Verlaufe der Behandlung keine erhebliche Besserung erfuhr. Bei mehreren Patienten, die während der Ohrenbehandlung mit der Zunahme des Gehörs gleichzeitig eine solche der Sehkraft erlangt hatten, blieb diese letztere auch späterhin unverändert, trotzdem in der Ohrenaffektion wieder eine wesentliche Verschlimmerung mit einer bedeutenden Herabsetzung des Gehörs eingetreten war.

Es könnte ferner vielleicht die Vermuthung aufgestellt werden, dass die Veränderungen des Sehvermögens auf Druckschwankungen der Labyrinthfliussigkeit beruhen, welche entweder dureh Lufteinblasungen in die Paukenhöhle oder, bei den verschiedenen Reizeinwirkungen auf das $\mathrm{Ohr}$, infolge einer dadurch herbeigeführten reflektorischen Contraction des Trommelfellspanners, möglicherweise zu Stande kommen.

Gegen eine solche, an und für sich schon unwahrscheinliche Annahme spricht die von mir beobachtete Beeinflussung des Sehvermögens durch mechanische Reizung des äusseren oder mittleren Ohres in Fällen von Luxation des Ambos-Steigbügelgelenkes, bei 
Ueber d. Einfluss von Trigeminus-Reizen auf d. Sinnesempfindungen etc. 165

denen also auch der Muse. tensor tympani den Labyrinthdruck nicht $z u$ ändern vermochte.

Da den angeführten Beobachtungen zufolge weder Veränderungen der Hörfähigkeit noch Schwankungen des Labyrinthdruckes als Ursachen der besprochenen Alterationen des Sehvermögens zu betrachten sind, so können diese wohl nur dadurch zu Stande kommen, dass bestimmte, sowohl im äusseren als auch im mittleren Ohre befindliche Nerven durch einen sie treffenden Reiz den Gesichtssinn auf dem Wege des Reflexes (im weiteren Sinne des Wortes) beeinflussen.

Es wäre hier zu bemerken, dass vom Ohre auslösbare Reflexerscheinungen am Bewegungsapparate des Auges bereits wiederholt constatirt wurden ${ }^{1}$ ).

Unter den das äussere und mittlere Ohr versorgenden Nerven (N. N. trigeminus, facialis, glosso-pharyngeus, vagus, sympathicus und rami plexus cervicalis) wäre betreffs einer Reflexeinwirkung auf den Lichtsinn vor Allem der Trigeminus in Betracht zu ziehen, da dieser Nerv einerseits sämmtliche Theile des äusseren und mittleren Ohres versorgt, von denen an den erwähnten Versuchspersonen ein Einfluss auf das Sehvermögen nachweisbar war und

1) So beobachtete Deleau (s. Schmidt's Jahrb. 1840, 2. Suppl.-Bd., S. 209) in einem Falle von eitriger Entzündung des mittleren Ohres mit Polypenbildung ein konvulsivisches Zucken des Auges, das sich nach Heilung des Ohrenleidens verlor. - Schwabach (Deutsch. Zeitschr. f. prakt. Heilk. $1878, \mathrm{Nr} .1$ ) bemerkte an einem Patienten, bei Druck auf die eitrig entzündeten Wände der Paukenhöhle einen Nystagmus, P flüg er (Deutsch. Zeitschr. f. prakt. Heilk. 1878, Nr. 35), nach Entfernung eines Ohrenpolypen, bilateral oscillatorische Augenbewegungen, Bürkner (Arch. f. Ohrenheilkunde, Bd. 17, S. 185) bei Einführung des Trichters in den äusseren Gehörgang, sowie bei Ausspritzung des Ohres, ebenfalls Nystagmus. An einem von mir beobachteten Mädchen war durch Eindringen eines Insektes ins $\mathrm{Ohr}$, eine eitrige Ohrenentzündung aufgetreten, während derer ein beiderseitiger, ausserordentlich starker Nystagmus (in einer horizontalen Richtung) entstand, der auch nach Ablauf der Ohrenentzïndung, 10 Jahre später, unverändert anhielt. Einwirkungen auf die Pupille und zwar Erweiterung derselben infolge von eitriger Entzündung der Paukenhöhle, fanden Moos (Arch. f. Ohrenheilk. Bd. 2, S. 200) und Schwartze (Arch. f. Ohrenheilk. Bd. 16, S. 263). Bei einer von mir behandelten Patientin war während der Extraction eines Paukenpolypen eine Ablenkung des Auges nach aussen eingetreten; der Strabismus hatte auch einige Monate später in gleicher Weise fortbestanden. 
andererseits die Erfahrung dafür spricht, dass unter allen sensiblen Nerven von den sensitiven Trigeminus-Aesten eine reflektorische Einwirkung auf das Centralorgan am leichtesten erfolgt.

Um zu erfahren, ob die Beeinflussung des Sehvermögens vom Ohre aus dureh die sensiblen Trigeminuszweige erfolge, stellte ich an verschiedenen Individuen Versuche an, inwiefern durch Reizung der ausserhalb des Ohres verlaufenden sensitiven Trigeminuszweige Veränderungen im Sehvermögen eintreten können.

1) Bij. Jaeger Nr. $8=0$, nach schwachem Anblasen der Wange $=0$ nach starkem Anblasen der Wange erscheint auf einige Sekunden das Wort "Die", so auch bei einem 2. und 3. Versuch.

2) Englinger. Nr. $17=0$; nach Anblasen der Nasenmuschel tritt das 1. Wort "Liebe" auf und geht nach einigen Sekunden wieder zurück.

3) Giwisch. L. A. Nr. 19=0. Nach Anblasen der linken Wange vermag die Versuchsperson das Wort "Hamburg“ deutlich zu lesen; im nächsten Augenblicke ist jedoch die Sehsteigerung wieder verschwunden.

4) Stieglitz, 14 Jahre. Am rechten Auge Nyopie ${ }^{16}{ }_{15}$, Sehschärfe ${ }_{1}$; am linken Auge Myopie $\left.{ }^{1}\right|_{18}$, Sehschärfe $\left.{ }^{1}\right|_{1}$; binoculäre Sehschärfe ${ }^{1}{ }_{1} ;$ Jaeger Nr. 1 v. $12^{\prime \prime}-3^{\prime \prime}$; mit dem Augenspiegel: Rechts Myopie ${ }^{1 / 16}$; links Myopie $\left.{ }^{1}\right|_{24}$; beiderseits die innere Sehnervengrenze verwischt, nach aussen beginnender Conus, rechts breiter als links (B e rgmeister).

Nach Anblasen der Nasenmuscheln hellt sich das Gesichtsfeld, 5 Sek. später, auf und wird nach 20 Sekunden wieder dunkler. Anblasung der Wange bewirkt eine vorübergehende schwache Aufhellung. Die Application eines Inductionsstromes, bei Anlegung der einen Elektrode an den Tragur, der andern an den Hals, erregt ebenfalls eine Aufhellung des Gesichtsfeldes. Beim stärkern Anblasen des Ohreinganges der rechten Seite hellt sich das Gesichtsfeld auch am linken Auge auf, dagegen ruft ein schwaches Anblasen der rechten Seite nur am rechten Auge eine Aufhellung hervor. An einem anderen Versuchstage ergibt das Anblasen der linken Wange am rechten Auge eine bedeutende Aufhellung des Gesichtsfeldes, nach 20 Sekunden erfolgt eine Abnahme derselben, nach weiteren 20 Sekunden zeigt sich das Gesichtsfeld so abgedunkelt wie vor dem Versuche; einige Sekunden später gibt sich wiederum eine vorübergehende Aufhellung zu erkennen. Es wird nunmehr dem linken Auge Jaeger Nr. 16 vorgelegt, von der nur die Worte "Finer" ... „ist". . hervortreten; unmittelbar nach Zuleitung von Ammoniakdämpfen in die linke Nasenseite liest das Versuchsindividuum von Nr. $16=$ Einer ... ist nichts ..., einige Sekunden später abermals nur: Einer ... ist ... Bei einem unmittelbar darauffolgenden 2. Versuche liest das linke Auge: Einer $n$. ist nichts .., 10 Sekunden danach nur: Einer ... ist... 
Ueber d. Einfluss von Trigeminus-Reizen auf d. Sinnesempfindungen etc. 167

Die Prüfung des rechten Auges ergibt folgendes: Nr. $16=$ Einer ... ist ...; nach Einleitung von Ammoniakdämpfen in die linke Nasenseite Nr. $16=$ Einer ... ist nichts $\ldots, 1$ Minute später $=$ Einer . . . ...h..t ist nichts ... Eine vergleichsweise Prüfung des rechten und des linken Auges weist anstatt des sonst vorhandenen identischen Sehvermögens nunmehr eine beträchtlichere Erhöhung der Sehkraft am rechten Auge nach; binnen 4 Minuten sinkt die Sehbesserung an beiden Augen anf das ursprüngliche Niveau herab. Das Versuchsindividuum behauptet bei allen Sehprüfungen stets zu bemerken, dass dem rechten Auge die Buchstaben deutlicher erscheinen, dem linken Auge dagegen das Papier der Schriftskala heller.

Dureh die angeftibrten Versuchsergebnisse ist der Nachweis erbracht, dass auch eine Reizung der, das Ohr nicht versorgenden, sensiblen Trigeminusfasern eine Steigerung des Lichtsinnes, bez. des Sehvermögens in ähnlicher Weise veranlassen könne, wie dies bei einer Irritation der Trigeminuszweige im äusseren oder mittleren Ohr der Fall ist. Beim Anblasen der Wange oder der Nasenschleimhaut, bei elektrischer Reizung, sogar beim Riechen scharfer Stoffe kann eine vorübergehende Steigerung des Lichtsinnes eintreten. Eine solche gibt sich bei den photometrischen Prüfungen in einer plötzlichen Aufhellung des Gesichtsfeldes zu erkennen, wobei, oft nur auf einige Sekunden, früher nicht sichtbar gewesene Streifen an der Photometertafel deutlich wahrnehmbar erscheinen. Bei Prüfung mit den Schriftskalen kann sich nach dem Anblasen der Wange, der Nasenschleimbaut ete. ${ }^{1)}$ entweder unmittelbar danach oder einige Sekunden später eine Aufhellung des Sehfeldes bemerkbar machen, wobei einzelne Buchstaben, mitunter einzelne Worte plötzlich deutlich hervortreten, jedoch meistens bereits nach einigen Sekunden wieder verschwinden. Eine auf das Anblasen der benannten Gesichtstheile durch Minuten anhaltende Sehsteigerung findet sich viel seltener vor als bei Lufteinblasungen ins Mittelohr und besonders bei Bougirung des Tubenkanales. In gleicher Weise ist auch der Reizeffekt beim Anlasen der Wange oder Nasenschleimhant meistens weit geringer wie bei den erwähnten Einwirkungen auf das Mittelohr. Ein schwaches Anblasen der Wange oder der Nase bewirkt bei manchen Individuen nur das erste mal eine unbedeutende Sehsteigerung

1) Bei einem Versuchsindividuum ergab auch das Anblasen der Nackengegend eine vorübergehende Sehsteigerung. 
und bleibt bei wiederholter Austibung dagegen vollständig wirkungslos; zuweilen findet selbst bei energischem Anblasen der verschiedenen Theile des Gesichtes ïberhaupt keine Reaktion statt. Bei einigen Versuchspersonen erregte das Anblasen sogar eine vorïbergehende Herabsetzung des Sehvermögens, darunter auch bei Individuen, bei denen an anderen Versuchstagen, nach der Erregung der Trigeminus-Aeste des Gesichtes, eine Steigerung der Sehkrạft deutlich nachweisbar war. Andererseits findet wieder bei besonders empfindlichen Personen auf eine einmalige Anblasung der Wange ete. durch einige Zeit eine abwechselnde Aufhellung und Verdunklung des Gesichtsfeldes statt. Betreffs der Beeinflussung des Sehvermögens beider Angen, bei einseitig gesetztem Reize, geben sich beim Anblasen des Gesichtes ganz die gleichen Erscheinungen zu erkennen wie bei der Einwirkung auf ein Ohr: während schwache Reize oder Reize bei schwer erregbaren Indiduen nur auf das Sehvermögen des Auges derselben Seite einwirken, erstreckt sich dagegen der Einfluss stärkerer Reize oder der Reize bei leicht erregbaren Personen auch auf das andere Auge.

Da durch alle hier angeführten Versuchsergebnisse der Einfluss des Trigeminus auf das Sehvermögen bez. auf den Lichtsinn ${ }^{1}$ ) sichergestellt erscheint, so dürfte in Berücksichtigung der schon früher geltend gemachten Gründe wohl die Annahme berechtigt sein, dass die bei Erkrankungen des äusseren oder mittleren Ohres

1) Die Beeinflussung des Lichtsinnes durch den Trigeminus bietet wohl für die Lehre der Reflexamaurose eine Stütze dar; wenigstens ist damit die Deutung gegeben, wieso Verletzungen des Supraorbitalnerven (s. Leber, Handb. d. Augenheilk. von Gräfe und $\mathrm{Sämisch,} \mathrm{Bd.} \mathrm{5,} \mathrm{Th.} \mathrm{5,} \mathrm{p.} 975$ ff.), Caries dentis, sowie Geschwülste, die den Verzweigungen des Trigeminus aufsitzen, eine mehr oder minder hochgradrige Amblyopie erzeugen können, die entweder nur auf der leidenden Seite oder aber auf beiden Seiten eintritt (l. c.). Merkwürdig ist die schon von Hippokrates angeführte Beobachtung, dass die infolge von Verletzung der Supraorbitalgegend eintretende Sehstörung, keineswegs unmittelbar nach dem Trauma erscheint, sondern erst allmählich später während des Vernarbungsprozesses (vgl. Bergmeister, Wiener Klinik, 1880, Heft 1 v. 2). - Bemerkenswerth ist ferner die Angabe von Bernard (Leçons sur la physiologie, II, p. 90, s. Sigm. Mayer in Handb. der Physiol. von Hermann, Bd. 2, Th. 1, p. 240), dass bei Thieren denen der nv. opticus durchschnitten war, nach Hornhautverletzungen die Erscheinungen der Photophobie vorkommen; Bernard bezieht dieses Phaenomen auf eine Reizung der sensiblen Trigeminus-Fasern durch Licht. 
Ueber d. Einfluss von Trigeminus-Reizen auf d. Sinnesempfindungen etc. 169

zuweilen auffällige Veränderung des Sehvermögens infolge des reflektorischen Einflusses auftreten könne, welchen die dureh die Ohrenerkrankungen affizirten sensitiven Ohräste des Trigeminus auf die optischen Centren zu nehmen vermögen.

Der Umstand, dass ein, anf den Trigeminus der einen Körperhälfte ausgeübter stärkerer Reiz, ja bereits ein leichter Druck auf das eine Auge, seine Wirkung auch auf das andere Auge auszuüben vermag, verdient wohl bei Beurtheilung mancher sogenannter sympathischer Augenaffektionen nähere Berücksichtigung.

Der Nachweis einer Beeinflussung des Gesichtssinnes durch den Trigeminus legt die Frage nahe, ob dem Trigeminus auch auf die übrigen Sinnesorgane eine ähnliche Einwirkung zukomme.

Hinsichtlich des Hörsinnes liegen bereits zahlreiche Beobachtungen vor, aus denen die Möglichkeit einer reflektorischen Beeinflussung der acustischen Centren rom Trigeminus-Gebiete aus klar hervorgeht ${ }^{1}$ ).

1) Fine Verminderung der subjektiven Gehörsempfindungen beobachteten Türk (Oest. Wochenschr. 1843, Nr.44, s. Canstatt's J. 1843, Bd. 3, p. 192) bei Ausübung eines Druckes auf die Stirne, den harten Gaumen und auf die Zunge, Wilde (Med. Times and Gaz. 1852, s. Schmidt's J., Bd. 76, p. 83) bei Reibung der Tragus-Gegend, Weil (Mon. f. Olrenhk. XI, XII, p. 68) beim Anblasen des äusseren Ohres. In zweien von mir behandelten Fällen wurden die vorhandenen subjektiven Gehörsempfindungen durch Aetzung der geschwellten Schleimhaut an der unteren and mittleren Nasenmuschel vollständig und dauernd zum Stillstand gebracht (s. mein Lehrb. der Ohrenheilk. 1880, p. 254). - Eine durch Irritation der sensiblen Trigeminus-Aeste bervorgerufene Erreguing von subjektiven Gehörsempfindungen fanden u. A.: Valleix (s. Schmidt's J. 1855, Bd. 85, p. 177) während eines Anfalles von Trigeminus-Neuralgie, Politzer (Wien. med. Wochenschr. 1865) bei Entzündung des äusseren Gehörganges, Schwartze (Berl. klin. Wochenschr. 1866, Nr. 12 u. 13) bei Dentalgie. Henle (s. Joh. Müller, Handb. d. Physiol. 1840, Bd. 2, p. 482) beobachtete an sich beim Reiben der Wange ein Rauschen im Ohr. In einem Falle von Zaufal (Wien. med. Wochenschr. 1872, Nr. 21) trat beim Streichen der Tragusgegend regelmässig die subjective Empfindung des Tones $\mathrm{e}^{\prime \prime \prime}$ auf. Benedikt (Wien. Wochenbl. 1863, Nr. 23) betrachtet die durch galvanische Reizung ausgelösten subjektiven Gehörsempfindungen als eine vom Trigeminus erregte Reflexerscheinung. - Als Beispiele einer Beeinflussung der Hörfunction vom Trigeminus aus, wären folgende anzuführen: Notta (Arch. gén. 1854, s. Schmidt's J., Bd. 85, p. 173) theilte einen Fall mit, in welchen während einer Trigeminus-Neuralgie Schwer- 
Hierher glaube ich, wenigstens zum Theile, auch den giinstigen Einfluss beziehen zu können, den Bougirungen der Ohrtrompete ohne nachfolgende Lufteintreibungen ins $\mathrm{Ohr}$ ausiuben, ferner den zuweilen plötzlich auftretenden günstigen Einfluss von irritirenden Flïssigkeiten oder Dämpfen ins Mittelohr. In ersterer Beziehung, wäre vor Allem anf die Thatsache hinzuweisen, dass, wie ich mich öfters uberzengt habe, durch eine einfache Bougirung des Tubenkanales eine Besserung der Sehwerhörigkeit und der subjectiven Gehörsempfindungen auch in Fällen eintreten kann, in denen der eventuell reflectorisch gereizte Trommelfellspanner keinen Einfluss auf den Labyrinthdruck (vermittelst der Kette der Gehörknöchelchen) auszuïben vermag, wie dies z. B. bei einer Luxation des Ambos-Steigbügel-Gelenkes der Fall ist. Auch die häufig zu beobachtende Erscheinung, dass sich die Empfindung von zunehmender Erleichterung des Kopfes und eine Abnahme des im Ohre vorhandenen Druckgefühles, sowie eine bedeutende Beruhigung der subjektiven Gehörsempfindungen und eine Hörbesserung, eben dann deutlich bemerkbar machen, während noch der Tubenkanal seitens der eingeführten Bougie vollständig verstopft ist, und dass die genannten Symptome um so auffälliger hervorzutreten pflegen, je dicker die Bougie ist, also je stärker sie auf die Wandungen des Isthmus tubae drickt, dürfte im obigen Sinne zu deuten sein.

Die zuweilen auffällige Einflussnahme, welche die verschiedenen Affektionen der Trigeminus-Zweige der einen Körperseite auf den Gesichtssinn der anderen Körperseite aufweisen, gibt sich in ähnlicher Weise an dem Gehörorgane in einer sympathischen

hörigkeit bestand; Lucae (Arch. f. Ohrenhk., Bd. 3, p. 227) beobachtete einen Fall von Schwerhörigkeit nach Dentalgie, Vautill (s. Wedel, Pathol. der Zähne, 1870) eine Trigeminus-Neuralgie mit Taubheit, die durch eine Extraktion des oberen letzten Mahlzahnes geheilt wurde. - Allerdings sind bei einer Trigeminus-Neuralgie die Symptome von Ohrensausen und Schwerhörigkeit nicht immer centralen Ursprunges, sondern beruhen u. a. möglicherweise auch auf einer reflektorischen Contraktion des Tensor tympani; wenigstens beobachtete Weber-Liel (Ueber d. progress. Schwerhörigk., 1873, p. 2) dass die während eines Migräneanfalles sonst regelmässig aufgetretenen Symptome von Schwerhörigkeit und subjektiven Gehörsempfindungen nach einer Durchschneidung der Sehne des Trommelfellspanners vollständig ausbleiben können. 
Beeinflussung des einen Ohres durch das andere Ohr ${ }^{1}$ ) zu erkennen; so kann beispielsweise bei einer ausschliesslichen Behandlung des einen Ohres eine Steigerung der Hörfähigkeit, sowie eine Verminderung der subjectiven Gehörsempfindungen auf dem anderen nicht behandelten Ohre erfolgen '). In einem von mir jüngst behandelten Falle mit rechtsseitiger Anaesthesia acustica für die Sprache und für verschiedene, den Kopfknochen aufgesetzte, stark tönende Stimmgabeln, waren durch die Bougirung des eatarrhalisch affizirten, verengten Tubenkanales am linken Ohre, die heftigen subjectiven Gehörsempfindungen am nicht behandelten rechten Ohre allmäblich zuriuckgegangen. Wie ich mich bei den anlässlich dieses Falles angestellten Versuchen überzeugte, können ein Druck auf den Tragus, ein Reiben desselben sowie ein Anblasen des äusseren Obres, vorhandene subjective Gehörsempfindungen nicht nur auf dem Ohre der betreffenden Seite (s. oben S. 169), sondern auch am anderen Ohre vorïbergehend vermindern, bez. sistiren ${ }^{2}$ ). Bezüglich des Einflusses des Trigeminus auf den Geschmackssinn habe ich bereits an anderer Stelle ${ }^{3}$ ) angeführt, dass bei ein-

1) Wharton Jones (cit. b. Frank, Ohrenheilkunde, 1845, p. 133); Weber-Liel (Mon. f. Ohrenh. VIII, Nr. 6); Urbantschitsch, Ohrenh. 1880, p. 419 u. 496 ; ferner Mon. f. Ohrenh. 1877, Nr. 8.

2) Ueber sympathische Affektionen am Gehörorgane liegen noch folgende Beobachtungen vor: Nagh Samuel (Troph. Nerv. 1860, p. 65-77) bewirkt an Kaninchen eine elektrische Reizung des Nerv. auriculo temporalis Trigemini der einen Seite eine hochgradige Entziundung der Ohrmuschel, der einige Tage später eine sympathische Entzündung der andern Ohrmuschel folgt. Berthold (Zeitschr. f. Ohrenh. X, p. 191) fand nach Durchtrennung der Trigeminus-Wurzel an der Med. oblongata, Entzündungserscheinungen im Cavum tympani nicht nur an der operirten, sondern in $3 / 5$ aller Fälle auch an der entgegengesetzten Seite. - Ruyter, Cl. Bernard und Callenfels (s. Callenfels, Zeitschr. f. rat. Med. 1855, N. F. Bd. 7, p. $173 \mathrm{ff}$.) berichten über gewisse Wechselbeziehungen, die sich an den Gefässen der rechten und linken Ohrmuschel nachweisen lassen. So kommt nach Callenfels die Gefässerweiterung beim Kneifen des einen Ohres auch am anderen Ohre vor; verminderte Blutzufuhr zu einem Ohre, steigert die Temperatur am anderen Ohre; dass ein Erblassen und eine Temperaturverminderung an dem einen Ohre am anderen Ohre eine Hyperämie und eine Temperatursteigerung hervorrufen, bemerkten bereits Ruyter und Bernard (l. c.). Mooren und Rumpf (s. Centralblatt f. d. med. Wissensch. 1880, Nr. 19) stellten ähnliche Beobachtungen an den Gefässen der Iris an.

3) Beobachtungen über Anomalie des Gesohmackes, der Tastempfin- 
fachem (nicht exsudativem) Catarrh der Paukenhöhle an dem geschmacksperzipirenden Gebiete der erkrankten Seite sehr häufig eine Verminderung der Geschmacksintensität stattfindet. Ich hatte dieselbe damals ausschiesslich als Druckerscheinung der, seitens der geschwellten Paukenschleimhant, affizirten Chorda tympani und des Plexus tympanicus aufgefasst, glaube jedoch auf Grundlage neuerer Beobachtungen eine solche Geschmacksherabsetzung, wenigstens zum Theile, als eine von Trigeminus ausgelöste Reflexerscheinung auffassen zu können. Wie mir nämlich diesbezügliche Versuche zeigten, vermag eine Reizung sensibler TrigeminusZweige (in Folge von Lufteinblasungen ins Ohr, Anblasen verschiedener Theile des Gesichtes a. s. w.), durch Erregung der Geschmackszentren, eine bestehende Geschmacksempfindung zu steigern oder vorher nicht wahrnehmbare Geschmacksempfindungen plötzlich über die Bewusstseinsschwelle zu heben ${ }^{1}$ ). Bei einigen meiner Versuchspersonen, denen ich eine äusserst schwach schmeckende Substanz in die Mundhöhle einführte, trat die betreffende Geschmacksempfindung erst nach einer Erregung sensibler Trigeminus-Zweige hervor. Wenn man irgend eine Geschmacksflissigkeit in den Mund nehmen lässt und durch Ausspülung des Mundes die Geschmacksempfindung vollständig zum verschwinden bringt, so kehrt dieselbe zuweilen in dem Augenblicke wieder zurïck, in dem ein Reiz auf einen sensiblen Trigeminus-Zweig stattfindet.

Aehnliche Beobachtungen lassen sich am Geruchsinn anstellen. In mebreren Fällen gelang es mir durch Erregung eines sensiblen Trigeminus-Zweiges eine Geruchsteigerung herbeizuführen; auch infolge forcirter Respirationsbewegungen durch die Nasenhöhle kann eine auffällige Steigerung der Geruchsempfindungen angeregt werden $\left.{ }^{2}\right)$. An einem Versuchsindividuum, das linkerseits ein

dungen und der Speichelsekretion in Folge von Erkrankungen der Paukenhöhle. Enke, 1876 , p. 40 ff.

1) Die Möglichkeit einer reflektorischen Beeinflussung der Geschmackscentren vom Trigeminus aus, darf wohl bei den Untersuchungen über die geschmackspercipirenden Nerven (sei es durch physiologische Experimente oder in pathologischen Fällen) nicht ansser Acht gelassen werden.

2) Hierher glaube ich auch die Beobachtung Politzer's (Lehrb. d. Ohrenheilk. 1882, p. 490) beziehen zu können, dass bei manchen Patienten mit chronischer Mittelohr-Eiterung, ohne nachweisbare Veränderung der Nasenschleimhaut, ein herabgesetzter Geruchsinn besteht. 
schwächeres Geruchsvermögen aufwies als an der rechten Nasenseite, erzielte ich durch Anblasen der linken Wange regelmässig auf 30-45 Sekunden ein Steigen des Geruchsinnes, so dass die betreffende Versuchsperson während der angegebenen Zeit beiderseits die gleiche Intensität der Geruchsempfindung aufwies; ganz denselben Effekt ergaben in einem anderen ähnlichen Falle mehrere, dem Riechversuche vorausgeschickte kräftige In- und Exspirationen darch die Nasenhöble der schwächer perzipirenden Seite. In einem Falle, in welchem seit 4 Jahren Anosmie bestand, gelang es mir durch Einführung einer Bougie in die vollständig normale Ohrtrompete der rechten Seite, anf 10 Minuten eine Geruchsempfindung an der rechten Nasenseite auszulösen; bei der darauffolgenden Bougirung des linken Tubenkanales kehrte ebenfalls durch mehrere Minuten das Geruchsvermögen zurïck, diesmal jedoch nur auf der linken Nasenseite.

Endlich habe ich betreffs der taktilen Empfindung der Haut zu erwähnen, dass mir seit Jahren der Einfluss von Mitttelohrerkrankungen auf die Empfindlichkeit der Ohrmuschel und auf die Umgebung des Ohres, zuweilen auch auf die Empfindlichkeit der Gehörgangswände aufgefallen war u. z. bei Patienten, an denen nicht die geringsten Veränderungen am äusseren $\mathrm{Ohr}$ bestanden, welche als Erklärungsgrund der mitunter beträchtlich herabgesetzten Hautempfindlichkeit hätten dienen können. Dass in solchen Fällen thatsächlich vom Mittelohr ans eine Beeinflussung der cutanen Empfindlichkeit stattfinde, beobachtete ich am deutlichsten an einem Patienten mit chronischem Mittelohreatarrh, der während einer zunehmenden Schwerhörigkeit eine beträchtliche Abnahme der Empfindlichkeit an der Ohrmuschel der erkrankten Seite bemerkte und später während einer Bougirung des bedeutend verengten Tubenkanales binnen wenigen Tagen "das taube Gefühl der Ohrmuschel" vollständig verlor, ohne dass jedoch damit gleichzeitig eine Besserung der Schwerhörigkeit eingetreten war. - An einem gegenwärtig noch in meiner Behandlung stehenden Knaben war infolge einer bilateralen eitrigen Entzündung der Paukenhöhle eine vollständige Sprachtaubheit zu Stande gekommen; im Verlaufe der Behandlung stellte sich allmählich ein theilweises Sprachverständniss ein und gleichzeitig damit traten einige, an dem Knaben vorher nicht bemerkbare Erscheinungen auf. Während nämlich der Patient bisher eine selbst seiner Umgebung auffällige Unem- 
174 Urbantschitsch: Ueber den Einfluss von Trigeminus-Reizen etc.

pfindlichkeit des äusseren Gehörganges gegen die, behufs Reinigung des Ohres, eingeführten Haarnadeln, Ohrlöffel ete. an den Tag gelegt hatte, zeigten sich nunmehr beide Gehörgänge ohne irgend welche nachweisbare Veränderungen äusserst empfindlich; es wurde ferner bemerkt, dass mit der Besserung des Ohrenleidens die vorher bemerkenswerthe Unempfindlichkeit gegen scharfe Gerüche oder stark schmeckende Speisen vollständig geschwunden war, so dass sich der Knabe u. A. entschieden weigerte, gewisse Speisen zu geniessen, die er vorher ohne Widerrede zu sich genommen hatte, während er wieder andererseits für Zucker, von dem er sonst selbst auf Zureden nichts geniessen wollte, jetzt eine besondere Vorliebe anfwies. Patient zeigte ferner die Gewohnheit alle Speisen zu riechen und wies davon die stärker riechenden zurïck, eine Eigenthümlichkeit, die an dem Knaben vorher noch niemals bemerkt worden war.

Mit Zugrundelegung der oben angegebenen Beobachtungen glaube ich diesen Fall so deuten zu können, dass durch die Behandlung des Ohres die daselbst befindlicheu sensiblen TrigeminusAeste beeinflusst wurden, welche ibrerseits wieder eine reflektorische Steigerung der Sensibilität der Haut, sowie der Geruchs- und Geschmacksempfindung ausgelöst hatten.

Eine Beeinflussung der verschiedenen Sinnesfunktionen durch Veränderungen im Mittelohre scheint keineswegs sehr selten vorzukommen, wenigstens beobachtete ich mehrere Individuen mit Mittelohraffektionen, welche an Seite des erkrankten bez. stärker affizirten Ohres über eine Abnahme des Sehrermögens klagten und bei denen ich an der betreffenden Körperseite auch eine Verminderung der taktilen Empfindlichkeit der Hant an der Ohrund Wangengegend, ferner einen schwächeren Geruch und Geschmack als an der anderen Körperseite nachweisen konnte.

Wenngleich über die Einwirkung der sensiblen TrigeminusAeste auf die einzelnen Sinnesempfindungen noch eingehende Untersuchungen nöthig sind, so glaube ich doch aus den mitgetheilten Versuchsergebnissen folgern zu dürfen, dass der Trigeminus auf sämmtliche Sinnesempfindungen einen Einfluss zu nehmen vermag und dass demzufolge durch eine Affektion irgend eines sensibeln Trigeminus-Gebietes alle Sinne in Mitleidenschaft gezogen werden können ${ }^{1}$ ).

1) Inwiefern die durch das Gehör vermittelten Farben- und Lichtem- 
Danilewsky: Ueb. d. Wärmeproduction u. Arbeitsleistung d. Menschen. 175

Die Resultate aller dieser Versuche weisen für die sensiblen Bahnen nach, was Exner für die motorischen Bahnen erwiesen hat, nämlich, dass der Ablauf von Erregungen im Innern des Centralnervensystems dadurch, dass andere Erregungen in dasselbe eintreten oder eingetreten sind, begünstigt werden könne ${ }^{1}$ ).

\section{Ueber die Wärmeproduction und Arbeitsleistung des Menschen.}

Von

\section{Dr. B. Danilewgky}

in Charkow.

Es ist offenbar ein physiologisches Postulat, dass die gesammte Wärmeproduction resp. die ganze Summe der entwickelten lebendigen Kräfte im thierischen Organismus lediglich auf gewissen chemischen Umwandlungen der Körperbestandtheile beruhe. Obwohl die Physiologie keine direkte unmittelbare Bestätigung dieses Grundsatzes bis jetzt aufweist, so dürfen wir, von dem Gesetze der Erhaltung der Kraft ${ }^{2}$ ) ausgehend, nichts desto weniger an seiner Richtigkeit nicht zweifeln. Daran in quantitativer Beziehung knüpft sich die Frage an, wie viel Wärme oder allgemeiner - wie viel gesammte lebendige Kraft producirt der Mensch täglich und auf welche Weise kann man ihre Grösse berechnen, ohne direkte calorimetrische Beobachtungen anzustellen. Hätten wir genauen Auf-

pfindungen (Nussbaumer, Mittl. d. ärztl. Vereins in Wien, 1873, Bd. 2, p. 49; F. Bleuler und K. Lehmann, "Zwangsmässige Lichtempfindungen durch Schall" etc., Leipzig 1881), ferner die bei Geschmacks- und Geruchswahrnehmungen eintretenden Farbenvorstellungen, sowie die Schallvorstellungen durch das Gesicht (Bleuler und Lehmann) hierher zu beziehen sind, vermag ich nicht zu entscheiden, sondern beschränke mich auf diese Erscheinungen hinzudeuter.

1) Exner, Pflüger's Arch. f. Physiol. 1882, Bd. XXVIII, p. 487.

2) Ueber die Gültigkeit dieses Gesetzes für die dynamischen Leistungen des Organismus s. meine "Thermodynamische Untersuchungen der Muskeln“, Dies Archiv, Bd. XXI, p. 109, 1880. 